\title{
A CLOSER EXAMINATION OF FACETS OF SOCIAL INTERACTIONS IN PREDICTING POSTTRAUMATIC STRESS DISORDER AND FACTORS THAT MODERATE THESE ASSOCIATIONS
}

\author{
by \\ Naomi Ennis \\ Honours Bachelors of Arts, University of Guelph, 2012 \\ A thesis \\ presented to Ryerson University \\ in partial fulfillment of the \\ requirements for the degree of \\ Master of Arts \\ in the Program of \\ Psychology
}

Toronto, Ontario, Canada, 2016

(C) (Naomi Ennis) 2016 


\section{Author's Declaration}

I hereby declare that I am the sole author of this thesis. This is a true copy of the thesis, including any required final revisions, as accepted by my examiners.

I authorize Ryerson University to lend this thesis to other institutions or individuals for the purpose of scholarly research.

I further authorize Ryerson University to reproduce this thesis by photocopying or by other means, in total or in part, at the request of other institutions or individuals for the purpose of scholarly research.

I understand that my thesis may be made electronically available to the public. 
A Closer Examination of Facets of Social Interactions in Predicting Posttraumatic Stress Disorder and Factors that Moderate These Associations

Master of Arts, 2016

Naomi Ennis

Psychology

Ryerson University

\begin{abstract}
Posttraumatic social interactions are among the most robust predictors of posttraumatic stress disorder (PTSD) following trauma, but social interactions have been widely defined and quantified in the literature. This study explored whether negative social interactions were more strongly associated with PTSD symptoms than positive interactions among adults recently exposed to a traumatic event, as well as factors that moderate these associations. Participants $(N=149)$ were assessed by the clinicianadministered PTSD scale and completed self-reported measures of social interactions and disclosure style. Only negative social interactions, specifically general societal disapproval and disapproval from family and friends, emerged as significant predictors of PTSD severity in a multiple regression analysis. Sex, trauma type, and dysfunctional disclosure style did not moderate relationships between negative social interactions and PTSD severity. Findings imply that negative social interactions may be more integral to trauma recovery than positive ones. Clinical implications are discussed.
\end{abstract}




\section{Acknowledgments}

First, I would like to thank my supervisor, Dr. Candice M. Monson for her continuous support. I am deeply grateful for the lessons I have learned from her and for her dedication to my development as a researcher. I would like to thank Dr. Tae Hart who played a key role in this thesis. I am incredibly grateful for Dr. Hart's constructive feedback and commitment to helping me throughout this process. As well, I would like to thank Dr. Janice Kuo for her time and feedback. I am also very thankful to Dr. Anne Wagner for her contributions to this study. In addition, I am greatly appreciative of my peers at Ryerson University, in particular my peers in the lab who contributed to this study and whose support has helped me to grow both academically and personally. Finally, I would like to thank my parents, Janice and Jon, for their encouragement and unwavering support. 


\section{Table of Contents}

Author's Declaration for Electronic Submission of a Dissertation .................................. ii

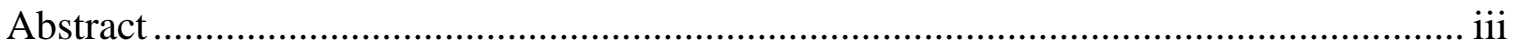

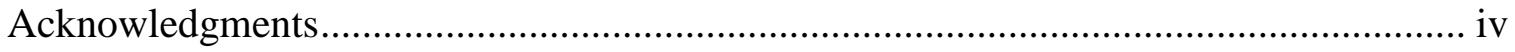

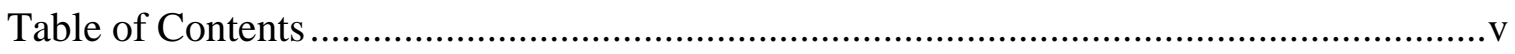

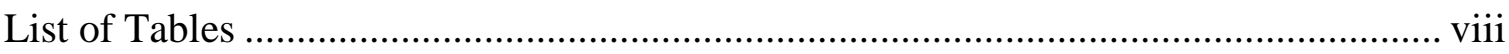

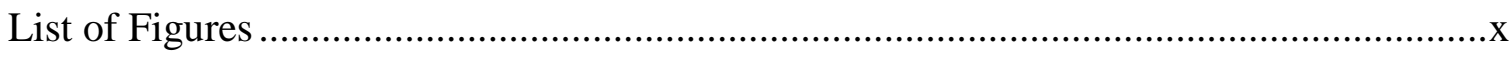

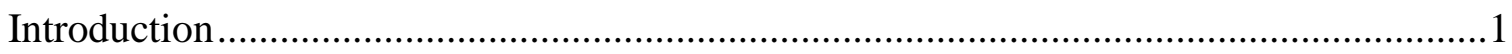

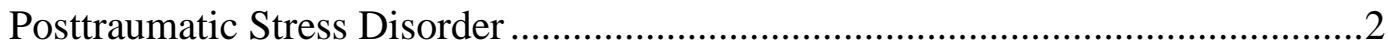

Risk and Resilience Factors for PTSD ....................................................

Defining Social Support: A Multidimensional Construct .................................6

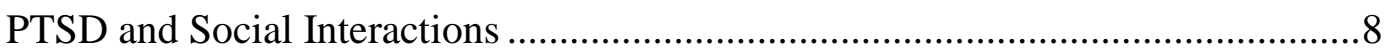

Facets of Social Interactions and Differential Effects on PTSD Symptoms .........11

Negative versus positive social interactions ............................ 12

Social Acknowledgment as a victim......................................15

Factors Associated with Social Interactions Following Trauma ........................19

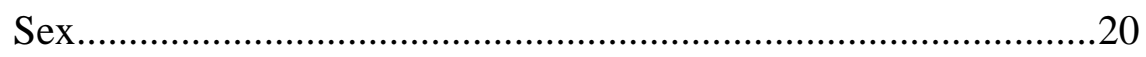

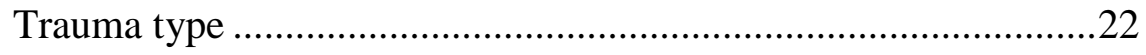

Disclosure of trauma style ..................................................23

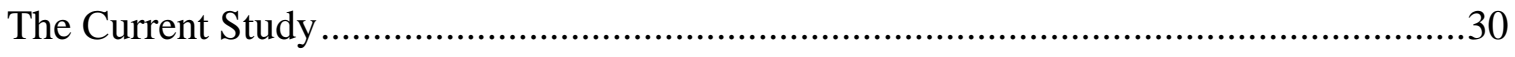

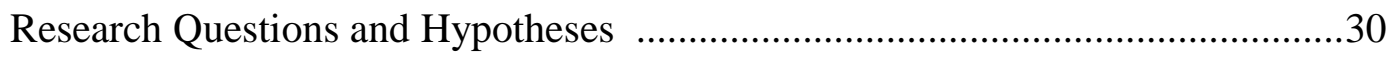

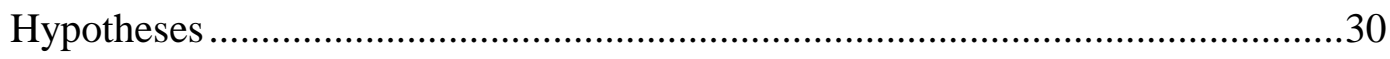

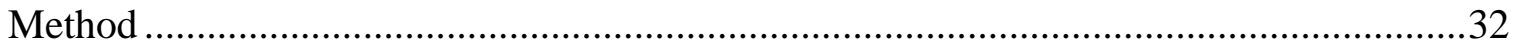




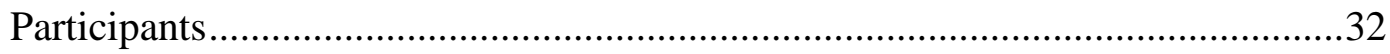

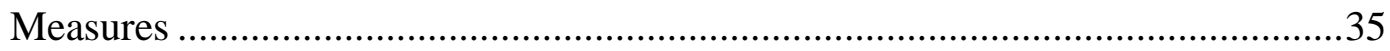

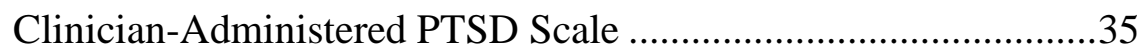

Social Reactions Questionnaire ............................................36

Social Acknowledgment as a Victim Questionnaire ....................38

Provisions of Social Relations Scale..........................................39

Disclosure of Trauma Questionnaire .....................................40

MINI International Neuropsychiatric Interview .......................41

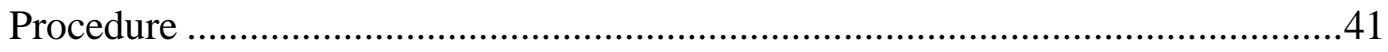

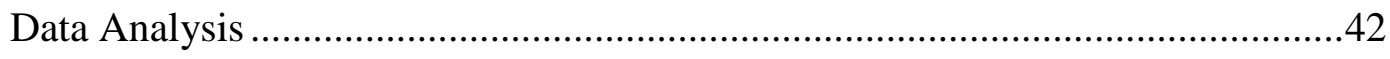

Hypothesis 1 ..................................................................4

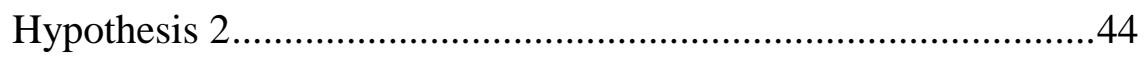

Model(s) for sex as a moderator ..................................45

Model(s) for disclosure style as a moderator ....................45

Model(s) for trauma type as a moderator.........................46

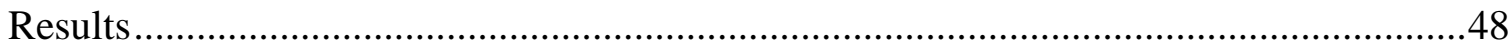

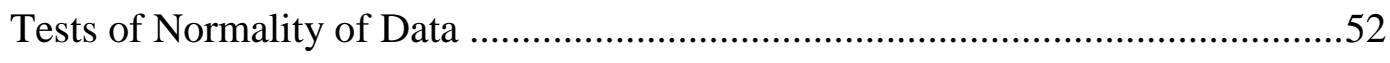

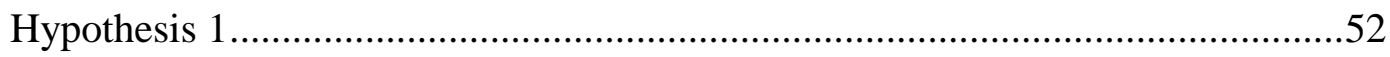

Bivariate Correlations with CAPS ........................................52

Regression Analysis ......................................................55

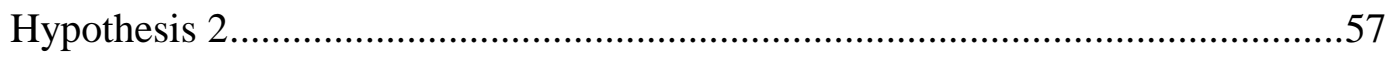

Models with sex as a moderator..............................................57

Models with disclosure style as a moderator .............................60 


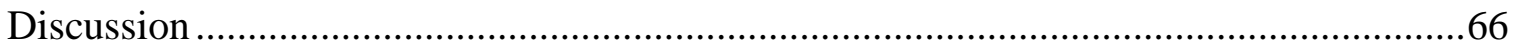

Negative Versus Positive Social Interactions: Aim 1 ………………..................66

Moderators of Relationships between Social Interactions and PTSD: Aim 2 ........71

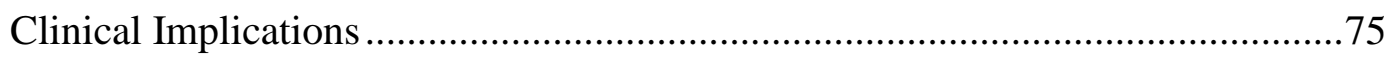

Limitations and Future Directions ....................................................................79

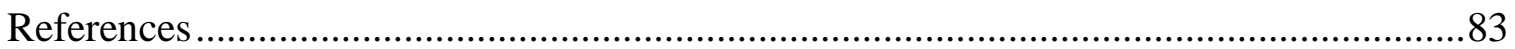




\section{List of Tables}

Table 1: $\quad$ Demographics of the Sample.

Table 2: $\quad$ Prevalence of Diagnostic and Statistical Manual of Mental Disorders, Fourth Edition, Text Revision Current and Lifetime Mental Health

Conditions among Participants

Table 3: Correlations between Social Interaction Scales, Disclosure Style, Age, Education, and Clinician-Rated Posttraumatic Stress Disorder

Symptom Severity

Table 4: $\quad$ Multiple Regression Analyses Predicting Clinician-Rated Posttraumatic Stress Disorder Symptom Severity from Negative, Positive, and General Measures of Social Interactions .56

Table 5: $\quad$ Sex as a Moderator of the Effect of General Societal Disapproval on Clinician-Rated Posttraumatic Stress Disorder Symptom

Severity .58

Table 6: $\quad$ Sex as a Moderator of the Effect of Family and Friends Disapproval on Clinician-Rated Posttraumatic Stress Disorder Symptom Severity 59

Table 7: $\quad$ Dysfunctional Disclosure Style as a Moderator of the Effect of General Societal Disapproval on Clinician-Rated Posttraumatic Stress Disorder Symptom Severity

Table 8: $\quad$ Dysfunctional Disclosure Style as a Moderator of the Effect of Family and Friends Disapproval on Clinician-Rated Posttraumatic Stress Disorder Symptom Severity. 
Table 9: $\quad$ Trauma Type as a Moderator of the Effect of General Societal Disapproval on Clinician-Rated Posttraumatic Stress Disorder Symptom

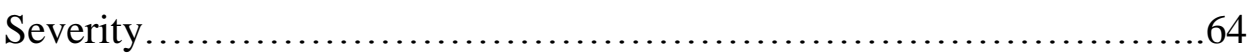

Table 10: $\quad$ Trauma Type as a Moderator of the Effect of General Societal Disapproval on Clinician-Rated Posttraumatic Stress Disorder Symptom

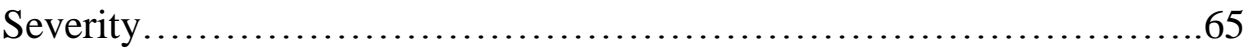




\section{List of Figures}

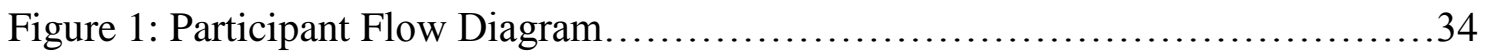




\section{A Closer Examination of Facets of Social Interactions in Predicting Posttraumatic Stress Disorder and Factors that Moderate These Associations}

Posttraumatic stress disorder (PTSD), a severe and chronic condition, is one of the most prominent mental health problems in Canada (Van Ameringen, Mancini, Patterson, $\&$ Boyle, 2008). At some point in their lifetime, $76 \%$ of Canadians will experience a traumatic event that could result in PTSD, but only $10 \%$ of those individuals will be diagnosed with the disorder (Van Ameringen et al., 2008). Unfortunately, for those who meet criteria for a diagnosis, the trajectory of PTSD is typically chronic and pernicious and is associated with significant personal and societal costs (Kessler, 2000; Van Ameringen et al., 2008). Yet, PTSD may be preventable if theoretically-driven early interventions are developed (Kessler, 2000). An understanding of risk and resilience factors for PTSD can help to inform such interventions.

Meta-analyses of risk factors for PTSD following trauma exposure indicate that posttraumatic social support is one of the most consistent and robust variables associated with the diagnosis (Brewin, Andrews, \& Valentine, 2000; Ozer, Best, Lipsey, \& Weiss, 2003). Therefore, early interventions that capitalize on social support may be a pathway to preventing PTSD. However, social support is a broad and multifaceted construct that can exist on a spectrum from positive (e.g., provision of emotional support) to negative (e.g., victim blaming, telling the victim to move on) interactions and can relate to individuals' experiences of support from different sources, such as acknowledgment of their experience as a survivor of trauma from members of their immediate social network or extend to include perceptions of society's treatment toward them as a victim (e.g., Helgeson, 2003; Maercker \& Mueller, 2004; Ullman, 1999). Researchers (e.g., Wagner, 
Monson, \& Hart, 2016) have recently called for the exploration of the specific facets of posttraumatic social interactions that most strongly relate to PTSD symptoms and the factors that are associated with an individual's likelihood of experiencing such interactions in the period acutely following trauma exposure. Knowledge of these factors is integral for the development of early interventions aimed at preventing or treating PTSD by enhancing interpersonal relationships and social support. Therefore, the objectives of the current thesis are twofold: 1) to examine the facets of social support (e.g. negative versus positive social reactions) most strongly related to PTSD symptoms; and 2) to explore the factors that are associated with trauma survivors' experiences of the different forms of posttraumatic social interactions most related to PTSD symptoms. In order to address these questions, a cross-sectional study of recently traumatized individuals (i.e., within 6 months of exposure to a traumatic event) exposed to various types of Diagnostic and Statistical Manual of Mental Disorders, Fourth Edition, Text Revision (DSM-IV-TR; American Psychiatric Association, 2000) Criterion A traumatic events was conducted.

\section{Posttraumatic Stress Disorder}

According to the Diagnostic and Statistical Manual of Mental Disorders, Fifth edition (DSM-5; American Psychiatric Association, 2013), PTSD is characterized by intrusion symptoms (e.g., flashbacks), persistent avoidance of stimuli associated with the traumatic event, negative alterations in cognitions and moods (e.g., persistent negative emotional state), and alterations in arousal and reactivity (e.g., sleep disturbances). As compared with other mental health problems, such as depression or schizophrenia, that develop over time or may have early warning signs, PTSD is considered a disorder of 
impeded recovery. For example, prospective studies indicate that acutely following trauma exposure, most individuals will experience an assortment of symptoms that can later be identified as PTSD symptoms if they have lasted for at least the one month period required for a diagnosis of PTSD as per DSM-5 diagnostic criteria (Ehlers, Mayou, \& Bryant, 1998; Riggs, Rothbaum, \& Foa, 1995; Rothbaum, Foa, Riggs, Murdock, \& Walsh, 1992). However, as illustrated by the findings that only $10 \%$ of trauma exposed individuals receive a diagnosis of PTSD, over time the majority of people experience abatement in their symptoms without any intervention, considered a form of "natural recovery" (Ehlers et al., 1998; Riggs et al., 1995; Rothbaum et al., 1992). Therefore, identifying the factors that impede the natural recovery of PTSD and put individuals at risk for PTSD in the period immediately following trauma exposure can help to inform early interventions aimed at preventing the disorder.

\section{Risk and Resilience Factors for PTSD}

Resilience, a term referring to protective factors that enable an individual to cope with adversity (Weiss, 2008), can be distinguished from risk, a term that refers to variables that increase an individual's likelihood of experiencing a certain outcome (Weiss, 2008). Although there is a growing body of literature on the risk and resilience factors associated with PTSD, studies vary greatly in terms of design, sampling, and measurement. For example, researchers have prospectively followed individuals after trauma exposure or compared those with PTSD to those without. Further, research has focused on different populations of trauma-exposed individuals such as combat veterans, victims of sexual assault, and survivors of natural disasters or accidents (Brewin et al., 2000). As well, different measures of PTSD symptom severity and risk factors have been 
used across the literature. Despite the great deal of heterogeneity across studies, posttraumatic social support has been identified as the most (Brewin et al., 2000) or among the most consistent and robust variables associated with the diagnosis of PTSD (Ozer et al., 2003) in meta-analyses of risk factors for PTSD.

Brewin and colleagues (2000) conducted a meta-analysis on 77 studies investigating populations exposed to trauma in adulthood and examined 14 risk factors. These risk factors included demographic factors (i.e., age, gender, socioeconomic status, education, and minority status), historical or static person characteristics (i.e., personal psychiatric history, family psychiatric history, intelligence, other previous trauma, childhood abuse, other childhood adversity), trauma severity, social support and current life stress. Peri- and posttraumatic variables such as trauma severity (weighted average $r=.23$ ) yielded larger effect sizes than pretrauma variables such as minority status (weighted average $r=.05$ ) and gender (weighted average $r=.13$ ), although all of these effects were statistically significant. Of these factors, poor posttraumatic social support emerged as the most potent predictor (weighted average $r=.40$ ). Although there was a stronger negative association between social support and PTSD for military as compared with civilian samples, posttraumatic social support still had the largest effect size across risk factors in both of these populations. Similarly, the relationship between PTSD and posttraumatic social support was not moderated by participant sex, timing of trauma (e.g., in childhood or adulthood), or study characteristics, such as retrospective or prospective design, assessments by interview or self-report questionnaire, and whether PTSD was measured by diagnosis or severity. 
Ozer et al. (2003) also conducted a meta-analysis of factors associated with a PTSD diagnosis, but in response to Brewin and colleagues' (2000) findings, only examined risk factors that were significant predictors of PTSD. Specifically, rather than focusing on demographic factors such as gender, education, and ethnicity, the focus was on individual characteristics that were salient for psychological processing and functioning related to aspects of the traumatic event or its sequalae. Seven predictors that had been sufficiently studied to warrant inclusion in the meta-analysis were explored, along with peritraumatic dissociation and peritraumatic emotional responses. In summary, factors explored were: 1) prior trauma, 2) prior psychological problems, 3) family history of psychopathology, 4) perceived life threat during the trauma, 5) posttrauma social support, 6) peritraumatic emotional responses, and 7) peritraumatic dissociation. All variables were found to be significantly associated with PTSD. However, peritraumatic dissociation was found to be the strongest predictor (weighted $r$ $=.35$ ), followed by posttraumatic social support (average weighted $r=-.28$ ). These findings suggest that in the studies reviewed, lower reported levels of perceived social support following trauma exposure were associated with higher levels of PTSD symptoms or rates of PTSD. Similar to Brewin et al.'s meta-analysis, the strength of the relationship between perceived social support and PTSD was not moderated by the type of sample studied or method used to assess PTSD symptoms or diagnosis.

Interestingly, Ozer et al. (2003) found that the inverse relationship between social support and PTSD was strongest among studies with more time elapsed between the traumatic event and PTSD assessment, and strongest amongst studies where more than 3 years had elapsed between the traumatic event and assessment of PTSD (average 
weighted $r=-.42$ ). In other words, in studies where more time had elapsed since the trauma, higher levels of perceived social support were associated with lower rates of meeting diagnostic criteria for PTSD and fewer PTSD symptoms. These findings suggest that any buffering effects of social support may be cumulative over time or that symptoms of chronic PTSD could potentially erode social support, pointing to the possibility that social support could be considered a risk factor, as well as a resilience factor. Importantly, this finding highlights the value of targeting social support as early on as possible following trauma exposure in order to establish beneficial patterns of social interactions for trauma survivors to prevent the onset or alleviate PTSD symptoms.

Ozer and colleagues (2003) also note that the measures used to evaluate social support in the included studies varied. For example, some evaluated the number of social network members giving support (e.g., Sutker, Davis, Uddo, \& Ditta, 1995), while others measured the availability of support from specific persons (e.g., Wolfe et al., 1998). However, most studies emphasized emotional support, highlighting that social support is a broad and multifaceted construct and the relationship between psychological stress and interpersonal resources is dependent on how social support is defined and measured.

\section{Defining Social Support: A Multidimensional Construct}

Although variably defined, social support most often refers to "[S]ocial interactions or relationships that provide individuals with actual assistance or that embed individuals within a social system believed to provide love, caring, or sense of attachment to a valued social group or dyad" (Hobfoll, 1988, p. 121). In a theoretical review paper of social factors and trauma recovery, Wagner and colleagues (2016) argue that social support is an umbrella term for a wide range of constructs. For example, they hold that 
the term social support has been used to refer to diverse constructs such as quantity of support (e.g., number of support providers or positive interactions), quality of support (e.g., perceived helpfulness of interactions), and objective or subjective experiences of support. Moreover, Wagner et al. describe that, typically in the literature, social support is examined and defined as positively valenced, suggesting that the reaction of others is at least perceived as helpful or supportive. However, Wagner and colleagues argue that often in the context of PTSD and other mental health conditions, social support is used to relate to a lack of support. Illustrated in Brewin et al.'s (2000) meta-analysis, lack of social support was more critical to the maintenance of PTSD than the availability of social support. For these reasons, researchers (e.g., Rook, 1984; Wagner et al., 2016) hold that the term social support as it relates to the social factors that influence PTSD symptoms is a "misnomer." Wagner and colleagues propose that the term "social interactions," provides a more precise, inclusive description of the social facets that play a role in mental health following trauma exposure, because interactions can also be negatively valenced referring to unsupportive, triggering, demeaning, or unhelpful reactions.

In their review, Wagner et al. (2016) found a growing body of literature demonstrating that negative interactions play at least as critical a role as those viewed as supportive in trauma recovery (e.g., Davis, Brickman, \& Baker, 1991; Ullman, 1999). For these reasons, Wagner et al. urge that to advance understanding of risk and resilience factors in trauma recovery, researchers must consider the full range of negative and positive social interactions as they relate to PTSD. The social environment is thought to influence how trauma survivors emotionally and cognitively process their traumatic 
experiences (e.g., Nietlisbach \& Maercker, 2009), and negative reactions to trauma survivors can promote maladaptive trauma-related cognitions that can contribute to PTSD symptoms (Charuvastra \& Cloitre, 2008). Several theories have been proposed to explain the relationships between PTSD and the posttraumatic social environment.

\section{PTSD and Social Interactions}

The most commonly cited models to explain the relationship between social factors and mental and physical health include the stress-buffering hypothesis, directeffects model, erosion of social support model, and the social negativity hypothesis. Different evidence supports each of these theories and depends on how social support is defined and measured. Cohen and Wills's (1985) "buffering hypothesis" is one of the most widely cited theories to explain the relationship between stress and health. The buffering hypothesis holds that supportive social networks help individuals to cope with stressful life events (e.g., interpersonal conflict, occupational stress) and that the stressbuffering effect of social support is influenced by the structure and function of the interpersonal resources used. In this model, structure relates to the quantity of support (e.g., number of supportive persons) and structure loosely refers to the quality (e.g., cooperativeness; Cohen \& Mannarino, 2008). The stress-buffering hypothesis holds that individuals with little or no social support who are experiencing stress will suffer from negative health effects, whereas for those with high levels of social support the deleterious effects of stress are reduced or eliminated.

The main- or direct-effects model, another theory to explain the relationship between social interactions and PTSD following trauma exposure, is a popular alternative to the stress-buffering hypothesis (Cohen \& Wills, 1985). This theory posits that social 
support is beneficial to health regardless of whether an individual is experiencing stress. In this theory, large social networks are thought to provide individuals with regular positive experiences and a set of defined social roles within the community that create a sense of stability and predictability for the individual. According to the model, social networks can also promote health by influencing health-related behaviours such as cigarette smoking, eating, and alcohol consumption or be related to health outcomes via emotionally induced effects on the neuroendocrine or immune system functioning. In this model, social support is defined as social embeddedness or social integration; that is, the variety of social relationships the person has and how embedded the person is in their social network (Cohen \& Wills, 1985). This theory posits that being well-integrated into a social network may shield an individual from having negative experiences (e.g., economic or legal problems) that could put them at risk of psychological or physical disorders (Cohen \& Wills, 1985).

Evidence for these different theories depends on how social support is measured and defined. For example, Cohen and Wills (1985) found that when studies used qualitative measures of social support to assess individual's perceptions of the availability of social support and others' responsiveness to their needs related to the stressful event, there was evidence to support the stress-buffering hypothesis. Alternatively, evidence for the main- or direct-effects model comes from studies that used measures evaluating an individual's degree of social integration in a large social network. These findings again highlight the broad and multifaceted nature of social support, emphasizing the importance of measuring different facets of social support when studying the relationship between psychological stress and social factors. 
More recently, researchers have explored an alternate hypothesis, that the symptoms of PTSD contribute to the erosion of social support over time (King, Taft, King, Hammond, \& Stone, 2006). In this framework, the symptoms associated with PTSD (e.g., social withdrawal, numbing, excessive anger) are thought to negatively influence the quality and quantity of social support received, rendering PTSD a risk factor for poor social support.

Along the same line, the social negativity hypothesis (Major, Zubek, Cooper, Cozzarelli, \& Richards, 1997) has been proposed to explain how social interactions following trauma exposure can also be a risk factor for mental health symptoms. Specifically, this theory posits that negative social interactions have a greater influence on adjustment and coping than positive social interactions, because people tend to give more weight to negative information in their judgments about a situation. In turn, these negative reactions can produce stronger adverse emotional reactions in trauma survivors. In this theory, social interactions can be considered a risk factor for PTSD, specifically negative social interactions (Major et al., 1997).

There is support for each of the aforementioned theories, illustrating the complex relationship between posttraumatic social interactions and PTSD and highlighting that social factors can pose as both risk and resilience factors. Indeed, longitudinal studies show that the directionality of the association between social support and PTSD varies over time (e.g., Kaniasty \& Norris, 2004; Laffaye, Cavellla, Drescher, \& Rosen, 2008), with early social support appearing to buffer against PTSD symptoms and PTSD symptom severity contributing to poor social support over time. Taken together, studies suggest that timing of social support is critical and underscore that early social support 
can serve as a protective factor, whereas PTSD symptoms serve as a risk factor for the erosion of support. However, as described, social support is multifaceted and echoing Wagner et al. (2016)'s theoretical review, the specific facets of social support most related to PTSD symptoms must be delineated in order to inform early interventions aimed at preventing and treating PTSD as to the social interactions that should be targeted acutely following trauma exposure.

\section{Facets of Social Interactions and Differential Effects on PTSD Symptoms}

Given the broad and multifaceted nature of social interactions, in their review, Wagner et al. (2016) argue that it is not surprising that the literature reveals that some social interactions, such as negative reactions to trauma disclosure (e.g., accusatory reactions) and experiences of family and general societal disapproval, relate more strongly to mental health symptoms following trauma exposure than others, such as positive reactions to trauma disclosure (e.g., comforting reactions) and perceptions of feeling supported in general (e.g., feeling close to family and friends). Indeed, traumaspecific support measures, such as scales assessing reactions to the traumatized individual (e.g., victim blaming, helping the victim get medical care) have been found to yield stronger relationships to PTSD symptoms than measures of general support, such as measures that can be used with any population (e.g., measures of feeling valued and cared for by members of one's social network; Maercker \& Mueller, 2004). As such, research has been directed to examining the perceptions of social interactions that are most integral to trauma recovery. Wagner and colleagues provide a review of recent literature investigating the relative importance of trauma-specific reactions from others, perceived as positive and supportive (e.g., supportive behaviours such as assisting the 
victim to get medical care) versus negative (e.g., victim blame, disbelief; Ullman, 1999), as well as the effects of these reactions from different sources of support, such as family, friends and society in general (e.g., Maercker \& Mueller, 2004). The current thesis aims to provide a more comprehensive review of this literature, as well as empirically explore the social factors most strongly related to PTSD following trauma exposure.

Negative versus positive social interactions. Studies consistently demonstrate that others' reactions to trauma disclosure experienced as unhelpful by victims are strongly correlated with PTSD symptoms following trauma, whereas there are mixed findings on the relationship between interactions perceived as positive and supportive with PTSD (e.g., Littleton, 2010; Ullman \& Filipas, 2001; Ullman, 1999). However, the majority of this literature has focused on sexual assault victims. For example, in a literature review conducted by Ullman (1999) to examine the differential impact of negative versus positive social reactions to disclosure of sexual assault, negative aspects of social relations (e.g., blaming the victim) most consistently demonstrated detrimental effects on victims, while there were mixed findings for the impact of relations experienced as positive. Some studies showed no effect, while others demonstrated a positive effect of perceived social support on posttrauma recovery for sexual assault victims. Ullman notes that only two studies explored negative social reactions and most studies did not use standardized measures of social support that capture the full range of supportive and unsupportive behaviours that can be experienced by victims. Ullman argues that more attention must be paid to negative social reactions. In response, Ullman (2000) developed the Social Reactions Questionnaire (SRQ), a measure to evaluate trauma survivors' perceptions of both negative (e.g., victim blaming, treating the victim 
differently, telling the victim to move on) and positive (e.g., providing emotional, instrumental, and informational support) social reactions to disclosure of trauma. This measure has subsequently received a great deal of popularity in the trauma literature.

The literature using the SRQ with sexual assault victims consistently demonstrates that negative social reactions are strongly associated with PTSD symptoms in both college and community samples of sexual assault survivors (Ullman, Filipas, Townsend, \& Starzynski, 2006; Ullman, Townsend, Filipas, \& Starzynski, 2007) and in cross-sectional (Ullman et al., 2006) and longitudinal (Littleton, 2010) studies. Again, findings are mixed as to whether positive social reactions to disclosure or behaviours perceived as supportive are predictive of fewer PTSD symptoms (e.g., Littleton, 2010). In a sample of 323 adult women who were sexually assaulted, Ullman and Filipas (2001) found that measures of current support and measures of assault-specific support were not related to PTSD symptom severity. However, negative social reactions, as measured by the SRQ, significantly predicted PTSD symptom severity, with being treated differently (e.g., stigmatizing responses) most strongly related to PTSD symptom severity. In contrast, a cross-sectional study of 699 female sexual assault survivors recruited from college, community and mental health agency sources found that positive social reactions and global social support as measured by the Social Activities Questionnaire of the Rand Health Insurance Experiment had significant, albeit small, effects in the positive direction on PTSD symptom severity (i.e., more positive social reactions and greater social support were associated with more PTSD symptoms; Ullman, Filipas, Townsend, \& Starzynski, 2007). Yet, among all the predictors explored, which included pre-assault (e.g., demographic factors), assault (e.g., perceived life threat) and postassault (e.g., avoidance 
coping, self-blame) psychosocial variables, negative social reactions were the second strongest predictors of PTSD symptom severity next to avoidance coping. Similarly, Littleton (2010) found that among 262 female college rape victims, both negative social reactions, as measured by the SRQ, and social support, measured by the Multidimensional Scale of Perceived Support, were moderate predictors of PTSD symptoms in cross-sectional analyses, with more negative social reactions predicting greater PTSD symptoms and greater perceived social support predicting fewer PTSD symptoms. At 6-month follow-up, only negative social reactions to disclosure predicted PTSD symptomatology, with increases in negative social reactions predicting greater PTSD symptoms. It is important to note that the positive social reactions scale of the SRQ was not used in this study (Littleton, 2010).

The relative influence of negative versus positive posttraumatic social interactions on PTSD symptoms has not been widely explored in other types of traumatized samples. One exception is a study by Andrews, Brewin, and Rose (2003), who assessed 118 male and 39 female survivors of violent crime (i.e., attempted physical or sexual assault or bag snatch) in England, with the Crisis Support Scale, a 6-item measure evaluating perceived social support and negative reactions. Only negative reactions and satisfaction with support were significantly correlated with PTSD symptoms. Specifically, negative reactions predicted greater PTSD symptoms and satisfaction with support was associated with fewer symptoms. Measures of social support (nontrauma specific measures of positive social interactions) did not yield any significant relationship with PTSD symptoms. This finding suggests that how one appraises the support one receives (i.e., their satisfaction with the support) may be more central to trauma recovery than the 
actual receipt of support. Similarly, in a longitudinal study by Dunmore, Clark, and Ehlers (2001) that assessed individuals who had experienced physical or sexual assault, negative perceptions of others' response to the trauma (i.e., "I feel like other people are ashamed of me now”) but not positive perceptions of responses (i.e., "There is somebody I can completely confide in,") were associated with PTSD symptom severity at 6- and 9month posttrauma assessments. Again, negative perceptions of others' responses were associated with greater PTSD symptoms.

Beyond assault victims, few studies have explored the differential impact of social responses to the victim (Guay et al., 2011). In Guay et al.'s (2011) sample of 96 individuals with a diagnosis of PTSD, the most common trauma was physical assault or threats (42\%) followed by vehicle accidents (26\%). Using the Questionnaire on Social Support Behaviours in Anxious Situations Questionnaire, a 31-item self-report questionnaire assessing the perceived frequency of supportive social interactions and negative social interactions, only interactions interpreted as negative were significantly correlated with PTSD symptoms. However, the mean time elapsed since trauma for the sample was 62.4 months $(S D=76.8)$ in their sample. A greater understanding of the relationship between social interactions and PTSD symptoms more proximal to exposure could better inform early interventions. As well, most studies have not explored PTSD with clinician-administered interviews, considered the gold standard in trauma research. Clearly, more research is needed on the relationships between negative and positive social interactions with PTSD symptoms among survivors of different types of trauma in the period acutely following trauma exposure using rigorous measures of PTSD.

Social acknowledgment as a victim. Based on findings that poor social 
integration, low social appreciation, rejection on homecoming and blaming reactions by their families and society at large are related to poor adaptation and PTSD symptoms in soldiers returning home (e.g., Solomon, Mikulincer, \& Flum, 1989), Maercker and Mueller (2004) assert that social acknowledgment as a victim is an important social construct related to wellbeing following trauma exposure. Social acknowledgment as a victim refers to the degree of validation and support trauma survivors experience from their social environment and has been defined as, “A victim's experience of positive reactions from society that show appreciation for the victim's unique state and recognition of the victim's current difficult situation (Nietlisbach \& Maercker, 2009, p. 385). Within this construct, "social" refers to both the closest members of the social network of a victim (e.g., family, friends) and also significant persons (e.g., local authorities, clergy), groups (e.g., at the workplace, fellow citizens), and impersonal expression of opinions (e.g., media) about the experiences of the victims (Maercker \& Mueller, 2004). "Positive acknowledgment" refers to unconditional support perceived by survivors, whereas in the negative case, victims experience a range of negative feedback such as rejection, victim blame, and feeling ignored (Maercker \& Mueller, 2004). This construct expands on typical facets of social support explored in the trauma literature to include the wider societal context. Social acknowledgment also differs from other facets of social support because it does not relate to the structural supportiveness or the functional supportiveness of the direct environment, but rather to a sense of having one's traumatic experience acknowledged and valued by members of one's social network and society at large. 
A growing body of literature points to the integral role of social acknowledgment as a victim in trauma recovery (Maercker, Povilonyte, Lianova, \& Pöhlmann, 2009). For example, a study of social factors related to PTSD symptoms in journalists who had covered the 2004 tsunami in Southeast Asia roughly 8 months following the event found that a low degree of social acknowledgment from supervisors and colleagues was significantly related to PTSD symptoms, whereas posttraumatic social support from family, friends, and colleagues was not (Weidmann, Fehm, \& Fydrich, 2008). In this study, social acknowledgment was measured with a single item about how much participants felt that colleagues and supervisors had acknowledged their work in the tsunami region.

In an effort to measure social acknowledgment with a standardized tool, Maercker and Mueller (2004) developed the Social Acknowledgment as a Victim Questionnaire (SAQ), a measure that has been found to be a better predictor of PTSD than conventional measures of perceived social support that are not trauma-specific and assess emotional support, instrumental support and social integration (Maercker \& Mueller, 2004). Unlike the SRQ, the SAQ also specifies the support source (i.e., family, general societal disapproval), allowing for comparisons between social acknowledgment from different sources. The SAQ has been found to be associated with PTSD symptoms in diverse samples of trauma survivors such as German crime victims (Mueller, Hanspeter, \& Maercker, 2008), former political prisoners (Maercker \& Mueller, 2004), refugees (Maercker et al., 2009), developmental aid workers (Jones, Mueller, \& Maercker, 2006), and individuals who have witnessed assisted suicide (Wagner, Keller, Knaevelsrud, \& Maercker, 2012). 
In line with findings from the SRQ, studies examining the SAQ among trauma survivors have found the subscales assessing negative social interactions, the general societal disapproval and family disapproval subscales, to be more strongly related to PTSD symptoms than the positively valenced scale, which measures societal recognition as a victim (Maercker et al., 2008; Maercker et al., 2009; Wagner et al., 2012). Moreover, general disapproval seems to most consistently correlate with PTSD symptoms. For example, in a longitudinal study of 86 German crime victims, general disapproval was significantly associated with PTSD symptoms at 5- and 11-months postcrime and family disapproval was significantly correlated with PTSD symptoms at 11-months postcrime. However, recognition was not significantly associated with PTSD symptoms at either 5or 11-months postcrime (Mueller et al., 2008).

In the validation study of the SAQ, the SAQ was administered to nontreatment seeking traumatized individuals in Germany, including 178 former political prisoners in East Germany and 151 recently traumatized interpersonal crime victims (Maercker \& Mueller, 2004). In this study, all three subscales were significantly correlated with PTSD symptoms; however, the family disapproval and general disapproval scales were more strongly positively correlated with PTSD symptoms, as compared with the recognition scale that was moderately negatively correlated with symptoms. Consistent with findings that general disapproval tends to be most strongly associated with PTSD symptoms, Jones et al. (2006) found that among 312 development aid workers, only the general disapproval scale of the SAQ was significantly correlated with PTSD symptoms, specifically only with intrusive recollections and hyperarousal symptoms. Family 
disapproval and recognition were not significantly related to any of the PTSD symptom clusters.

Taken together, findings from studies using the SAQ suggest that low societal appreciation may have the strongest association with PTSD symptoms. However, more research is needed with individuals who have been exposed to different types of trauma. As well, no identified studies have compared the SRQ, SAQ, and measures of general social support in a sample of survivors of diverse types of trauma acutely following trauma exposure. These studies are necessary to inform early interventions of the relative influence of the specific forms of social interactions that are most strongly associated with PTSD symptoms. Although societal acknowledgment as a victim and social reactions to disclosure are two of many social factors that could be explored among traumatized individuals, these constructs have emerged as particularly important because they are potentially modifiable in treatment (Xu et al., 2015). As well, these constructs are broad, encompassing positive social experiences such as emotional support (i.e., feeling comforted following trauma disclosure) and instrumental support (e.g., having a loved one assist in seeking medical care following trauma disclosure), as well as negative social experiences (e.g., feeling blamed or misunderstood) that individuals may report following trauma exposure. Given that these constructs allow for a parsimonious way of exploring traumatized individuals' social interactions, the current study focused on the relationships between these facets of social interactions and PTSD symptoms.

\section{Factors Associated with Social Interactions Following Trauma}

Although the literature points to strong associations between social facets and PTSD following trauma exposure, particularly negative social reactions to disclosure and 
social acknowledgment as a victim, little is known about the factors that predict whether a trauma survivor will experience such social interactions. Wagner and colleagues (2016) call for a better understanding of the variables that may moderate the relationships between specific social factors and trauma recovery. Knowledge of the factors that predict whether individuals will experience these different types of social interactions following trauma exposure is essential for building early interventions that aim to enhance social interactions and capitalize on an individual's social networks in an effort to prevent or treat PTSD symptoms. Research in this area is scarce, but Wagner et al. suggest that demographic factors such as the sex of the traumatized individual, peritraumatic factors such as type of trauma, and posttraumatic factors such as trauma disclosure may be associated with the likelihood of experiencing different forms of social interactions following trauma exposure. The following section offers an in-depth review of the literature on these potential moderators.

Sex. In Brewin et al.'s (2000) meta-analysis, civilian women were found to have a higher risk for PTSD than men, and researchers have posited that the higher risk of PTSD might be due to differences in the levels of social support experienced by men and women following trauma (Andrews et al., 2003). Yet, few studies have explored differences in the sex of trauma survivors with regard to posttraumatic perceptions of social support.

Andrews et al. (2003) explored sex differences in perceptions of posttraumatic social support among 118 male and 39 females who had survived a violent crime in England within the month preceding the study and at 6 months following the trauma. To measure social support, the Crisis Support Scale, which consists of six items relating to 
the perceived availability of others, confiding in others, emotional support, practical support, negative response of others and satisfaction with support was used. There were no significant differences between men and women's reports of having received positive support and their satisfaction with the support. However, women were more likely to report negative responses from family and friends, and the difference in level of negative responses predicted the sex difference in PTSD symptoms at 6-month follow-up, with higher rates of PTSD found among women versus men. Interestingly, the difference in level of negative responses from others between men and women was not explained by other demographic factors or crime characteristics (e.g., sexual or nonsexual crime). However, this study only used a single item to assess negative responses from others. DeLong (2013) assessed 200 men and women with a primary diagnosis of PTSD using the SRQ, the Inventory of Socially Supportive Behaviours, a 40-item measure evaluating objective levels of support, and the Social Support Questionnaire, a 17-item measure evaluating the number of perceived social supports in a person's life. Unlike Andrews and colleagues' study of violent crime survivors, participants in DeLong's sample had experienced a range of traumas including adult sexual assault (30.9\%), childhood sexual assault (17.8\%), adult nonsexual assault (22.0\%), accident (motor vehicle, or natural disaster; $14.1 \%)$, childhood nonsexual assault (6.8\%), death of or violence towards a loved one (5.8\%), and military combat $(2.6 \%)$. Women reported significantly lower objective levels of support, fewer positive social interactions, and had significantly less support available compared with men (DeLong, 2013). In contrast to Andrew et al.'s findings, no significant differences were found between men and women in negative social reactions. It is important to note that the type of trauma experienced 
and participant sex may be have been confounded in DeLong's study of mixed trauma survivors, although this was not examined in the study. For example, more women may have experienced sexual assaults and this type of trauma may be more likely to elicit fewer positive social interactions than types of traumas that men may have experienced. Although Andrews et al. did not find crime characteristics to explain the sex differences in negative responses experienced by participants, all participants in their study were victims of violent crimes. More research is needed to explore the interaction between sex and different types of trauma such as accidental traumas, sexual and nonsexual assault, and witnessing the death of a loved one with regard to social interactions, because type of trauma may be an important predictor of social interactions following trauma exposure.

Trauma type. Few studies have explored whether interpersonally-perpetrated traumas are associated with more negative social interactions than accidental traumas such as natural disasters or motor vehicle accidents. Most of the literature to date has focused on differences in social reactions among survivors of assault or violence. Early work has demonstrated that women disclosing sexual assaults report more negative social reactions than women who disclose nonsexual assaults (Baker, Skolnik, Davis, \& Brickman, 1991; Davis \& Brickman, 1996). However, it is important to evaluate whether there are differences in social interactions among those who have experienced different types of traumas, such as accidental versus interpersonal or sexual versus nonsexual traumas. Findings from such evaluations are necessary to determine if early interventions should be targeted toward certain types of trauma survivors.

In one of the only studies to compare social reactions among survivors of different types of trauma, Delong (2013) compared those who had experienced childhood 
sexual abuse to those who had not in their sample of participants, described earlier, who had experienced diverse traumas. Those who had experienced childhood sexual abuse reported significantly more negative social reactions than those who did not experience childhood sexual abuse. However, those who had experienced adult sexual assaults were not compared to those who had experienced nonsexual assault or traumas that were not interpersonal in nature (e.g., natural disaster, motor vehicle accident). Clearly, more research is needed to compare the social interactions of those who have experienced different types of traumas in order to identify those who may benefit most from early interventions targeted toward enhancing positive social interactions and reducing negative ones.

Disclosure of trauma style. There is a growing body of literature examining the relationship between trauma disclosure and the social interactions that trauma survivors experience (e.g., Maercker \& Mueller, 2004; Ullman \& Filipas, 2005; Ullman, 2008). For example, in their sample of 76 U.S. Air Force service members and their partners, Balderrama-Durbin et al. (2013) found a significant negative relationship between intimate partner support and PTSD symptoms, whereby greater intimate partner support was predictive of fewer PTSD symptoms. Interestingly, service members' willingness to disclose combat and deployment-related experiences accounted for most of the variance in the relationship between partner support and PTSD symptoms such that after controlling for partner support, the direct effect of partner support on PTSD symptoms was no longer significant (Balderrama-Durbin et al., 2013). Mediation analyses demonstrated that greater disclosure predicted fewer PTSD symptoms, and higher perceived partner support predicted greater disclosure. This finding highlights that 
disclosure may play an integral role in explaining the relationship between social support and PTSD.

The psychological processes involved in trauma disclosure and subsequent social interactions have been found to be highly intercorrelated (e.g., Maercker et al., 2009). For example, in a sample of 733 college students who had survived childhood sexual abuse, Ullman and Filipas (2005) assessed several aspects of disclosure including whether the participant had disclosed about the abuse they experienced, whether the disclosure was accidental or purposeful, age when they first disclosed, and timing of the disclosure (i.e., how long after the experience they first disclosed or someone found out). Disclosing sooner after the abuse was associated with greater negative social reactions as evaluated by the SRQ, whereas greater extent of disclosure was related to fewer positive reactions. These results suggest that certain styles of disclosing about the traumatic event may lead to adverse social interactions (Ullman \& Filipas, 2005). However, this study did not use a validated measure to evaluate disclosure characteristics.

Mueller, Beauducel, Raschka, and Maercker (2000) developed the Disclosure of Trauma Questionnaire (DTQ) to evaluate attitudes and styles of disclosing about a traumatic event, a measure that has also been considered to evaluate dysfunctional trauma disclosure style (Pielmaier \& Maercker, 2011). The questionnaire measures different aspects of trauma disclosure, including a victim's urge to talk about the event, reluctance to talk about the event, and emotional reactions while disclosing (Mueller et al., 2000) The DTQ scales have been found to be highly correlated with scales on the SAQ, suggesting that the way in which individuals communicate about their trauma is related to their experiences of posttraumatic social acknowledgment. In particular, research tends to 
demonstrate a positive relationship between the DTQ subscales and the SAQ subscales that assess negative aspects of social interactions in that the more one endorses dysfunctional disclosure styles, the more likely one is to experience negative forms of societal acknowledgment as a victim, such as family and general disapproval. Importantly, the family and friends disapproval and general disapproval scales have been found to be most strongly associated with PTSD symptoms (e.g., Mueller et al., 2008). The DTQ scales tend to have the opposite relationship with the aspect of social acknowledgment that relates to positive social experience, recognition as a victim, a measure of one's experience of feeling recognized and appreciated as a victim of trauma (e.g., Mueller et al., 2008). That is, the more an individual engages in dysfunctional communication styles (e.g., feeling an urge to constantly talk about the trauma, avoiding talking about the trauma, physical and emotional reactions while disclosing) the less likely they are to feel that their experience as a victim is recognized and validated by their social networks.

Findings from Mueller et al.'s (2008) longitudinal study of German crime victims supports that more dysfunctional disclosure styles are related to more negative and fewer positive social experiences following trauma exposure. Specifically, Mueller and colleagues found that the DTQ reluctance to talk scale was significantly moderately positively correlated with the SAQ general disapproval and family and friends disapproval scales and negatively correlated with the SAQ recognition scale. This finding indicates that an ambivalence to talk about the trauma may be related to perceptions of greater disapproval from family and society and more lack of recognition about the trauma. Similarly, the DTQ urge to talk scale was significantly positively correlated with 
the family disapproval and general disapproval subscales, but not the recognition subscale, the positively valenced measure of social acknowledgment as a victim. This finding suggests that the more one feels an urge to talk about one's trauma, the more likely one is to report general and family disapproval. The DTQ subscale assessing the traumatized individuals' emotional reactions while disclosing had a strong, positive, statistically significant association with the SAQ general disapproval scale. Again, this finding is noteworthy in light of the previous research that suggests a strong association between the general disapproval scale and PTSD symptoms, possibly suggesting that physical and emotional reactions while disclosing may be highly related to the experience of societal disapproval, a perception that has been found to be strongly associated with PTSD. Mueller and colleagues' study provides further evidence that dysfunctional disclosure styles, as measured by the DTQ, predicts more negative experiences of societal acknowledgment as a victim, such as family and general disapproval, and less positive social experiences, such as recognition as a victim, with general disapproval emerging as the facet most strongly associated with dysfunctional disclosure style.

Similar results come from Maercker et al.'s (2009) study of the role of social acknowledgment in PTSD symptoms among 61 Chechen refugees living in camps in Ingushetia. In this study, the SAQ subscales that are negatively poled, the general disapproval and family disapproval subscales, were generally significantly positively correlated with the DTQ subscales, again demonstrating that dysfunctional disclosure style is related to more negative social experiences (Maercker et al., 2009). In contrast, the positively poled SAQ scale, recognition as a victim, tended to be negatively correlated with the DTQ subscales, illustrating that more dysfunctional disclosure styles 
may be related to less positive social experiences following trauma exposure.

Specifically, this study found that more emotional reactions while disclosing predicted greater family disapproval and general disapproval and fewer feelings of recognition as a victim, as measured by the SAQ. The study also found that the more one feels an urge to talk about one's trauma, as measured by the DTQ urge to talk scale, the more likely one is to report family and general disapproval and the less likely they are to report feeling recognition for their experience as a victim. Finally, the study found that the more reluctant one is about talking about one's trauma, as measured by the reluctance to talk DTQ scale, the more likely one is to report family disapproval but not general disapproval. The findings of this study further illustrate that dysfunctional disclosure styles, such as emotional reactions while disclosing, having an urge to talk about the trauma, and reluctance to talk about the trauma, may be related to more negative social experiences following trauma exposure and less feelings of being recognized and appreciated as a victim.

These studies demonstrate that how one communicates about one's traumatic event may be strongly associated with the social reactions one experiences. However, it is important to acknowledge that the direction of this relationship is unclear because the majority of the research is correlational and one's perception of how supportive one's social network is may influence one's style of disclosure. The relationship between disclosure style and social interactions is likely reciprocal because the reactions one experiences subsequent to disclosure may influence how an individual communicates about the trauma. For example, an individual who receives negative reactions to disclosure may be more reluctant to disclose later on or may have more emotional 
reactions in subsequent disclosures. Although there have been studies examining the interrelationships between the DTQ and the SAQ, no research to date has examined how aspects of disclosure as measured by the DTQ relate to social reactions to disclosure, as measured by the SRQ. Understanding the styles of trauma disclosure associated with negative or positive social reactions to disclosure are critical for informing early interventions for PTSD aimed at enhancing social interactions.

This review demonstrates that social factors play an integral role in the mental health of trauma survivors and that some facets of social experiences may be more strongly associated with PTSD symptoms following trauma exposure than others. Specifically, negative reactions to trauma disclosure (e.g., blaming the victim) and experiences of general disapproval (e.g., feeling that the people where one lives respect them less after the incident or that important public figures in one's life did not express enough sympathy for what happened to them) may be most strongly related to experiencing PTSD symptoms following trauma exposure. In contrast, experiences of positive reactions from others following trauma disclosure (e.g., helping the victim access medical care), feeling recognized and appreciated as a victim by society, and feeling generally supported (e.g., experiences that are not trauma related such as the general feeling of being close to one's social network) have been found to be less strongly associated with PTSD symptoms in traumatized individuals. Given that negative social experiences may be more strongly associated with PTSD symptoms as compared with general or positive experiences of social support, it is important to examine the factors that may place an individual at risk of experiencing such negative interactions. This review suggests that female sex, having experienced a sexual trauma, and endorsing 
dysfunctional styles of communicating and disclosing about the trauma may be factors associated with experiencing such negative social interactions following trauma exposure. In their review, Wagner and colleagues (2016) call for additional research on the specific factors of social interactions most strongly related to trauma recovery and the factors that moderate these associations. The current study aimed to empirically explore these social factors and moderators in a sample of adults recently exposed to a traumatic event. 


\section{The Current Study}

\section{Research Questions and Hypotheses}

The current cross-sectional study sought to address gaps in the literature by examining the following questions in a diverse sample of individuals recently (i.e., within 6 months prior to study enrollment) exposed to different types of traumatic events (i.e., accidental, sexual, other interpersonal, and deployment-related events) using a goldstandard clinician-administered measure of PTSD: 1) What aspects of social interactions are most strongly associated with PTSD symptoms in recently traumatized individuals? Specifically, are negative social interactions more strongly associated with PTSD symptoms acutely following trauma exposure than positive social interactions and general perceived social support (i.e., non trauma specific measures of social interactions)?; and 2) Using findings from question 1, are there factors that moderate the association between the social interactions most strongly related to PTSD symptoms following trauma exposure?

Hypotheses. Based on the literature, the following hypotheses were made:

1) Negative social interactions will predict greater PTSD symptoms after controlling for positive social interactions and general social support. Specifically, negative reactions to disclosure and experiences of general disapproval will predict PTSD symptoms above and beyond positive social interactions and general social support, emerging as the only significant predictors of PTSD symptoms.

2) The relationship between negative social interactions and PTSD symptoms will be moderated by sex, trauma type, and disclosure style. Specifically, negative social 
interactions are expected to predict greater PTSD symptoms for females, those who have experienced a sexual trauma, and those who report dysfunctional disclosure styles. 


\section{Method}

\section{Participants}

The current study was part of a larger longitudinal research project exploring the trajectory of recovery acutely following trauma exposure. A sample of adults who were exposed to a DSM-IV-TR PTSD Criterion A traumatic event within the prior 6 months were recruited from various communities in the Greater Toronto Area using flyers, newspaper advertisements, and online advertisements. Advertisements were posted at university campuses, community centres, religious centres and hospitals with trauma centres. In addition, participants were recruited through clinician referrals from two sexual assault clinics at urban hospitals.

Interested participants made initial contact with the research personnel to receive additional information about the study and were screened over the telephone to assess for eligibility. Participants who met the following criteria were included in the study:

1) between the ages of 18 and 75;2) experienced a DSM-IV-TR criterion A trauma within the last 6 months; and 3) willing to consent to the study. In the parent study, there was an additional eligibility requirement that the traumatized individual participate with a close other who knew about the trauma. The term "close other" was meant to be as inclusive as possible and referred to a person with whom the interested participant had a close relationship. Close others could include, but were not limited to, intimate partners, close friends and family members. However, a subsample $(n=35)$ of those interested in participating in the study who met all other eligibility criteria did not participate with a close other. These individuals reported the following reasons for not participating with a close other: a close other was not available to participate (e.g., did not have time 
available) or willing to participate in the study $(n=10)$; there was no one in their life who they considered a close other $(n=10)$; they did not want to disclose their trauma to another person $(n=4)$; and they preferred to participate as a non dyad or they were not comfortable asking another person to participate with them $(n=4)$. For seven participants, the reasons for participating alone were not known. In order to make the inclusion and exclusion criteria as unrestrictive as possible to increase the external validity of the study, the criterion of having to participate with a close other was waived for these individuals but interested participants were strongly encouraged to try to identify a close other who could participate with them. Each member of the dyad completed the assessments individually. For the purposes of the current study, only data from the traumatized individuals were examined.

An initial sample of 162 participants met eligibility for study participation and, after accounting for participant dropout and partial completion of assessments, a final sample of 149 individuals was included in the current study. Figure 1 depicts the recruitment flow. 


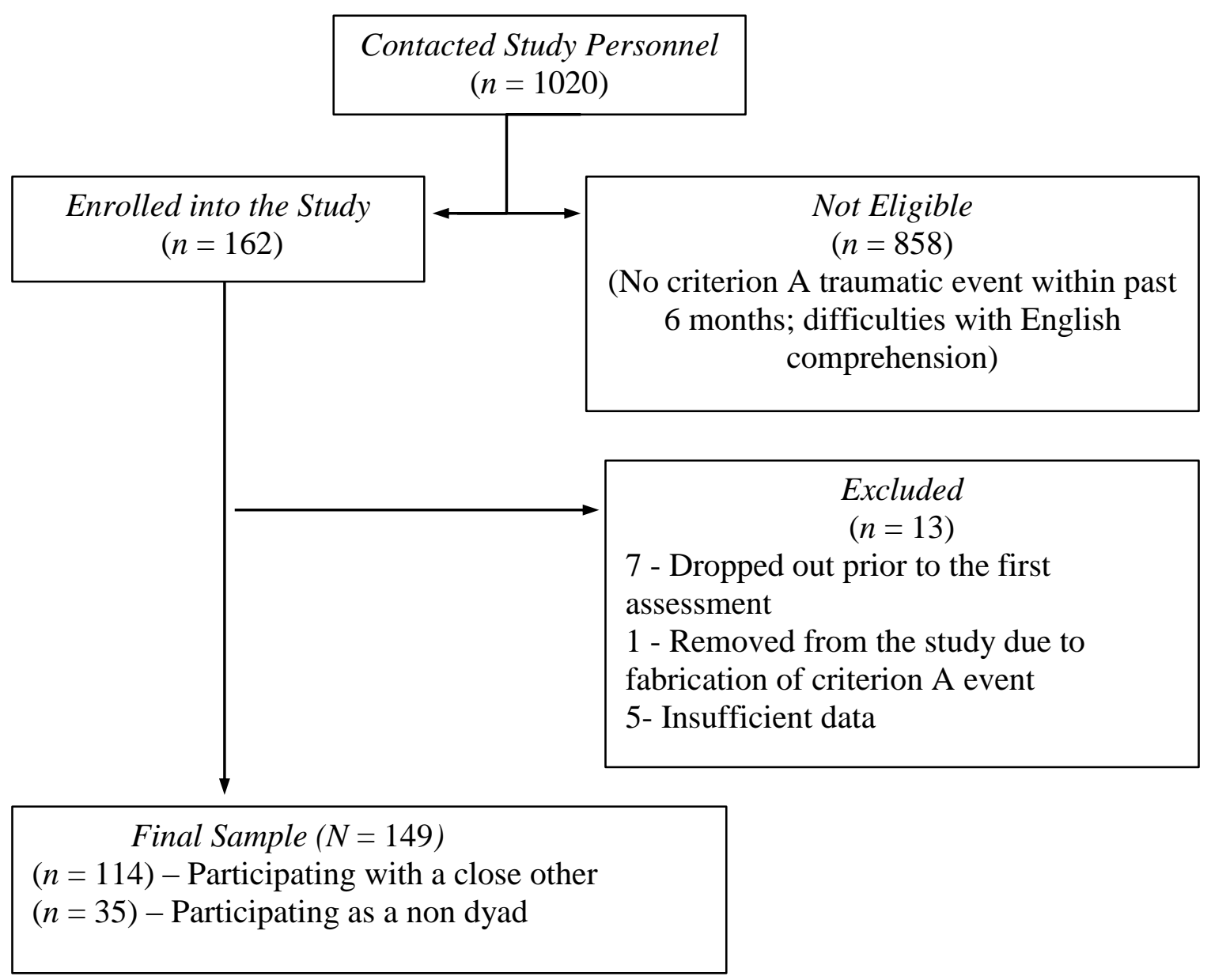

Figure 1. Participant flow diagram. 


\section{Measures}

Participants were interviewed using semi-structured assessments administered by graduate-level clinical psychology students. In addition, participants completed a package of self-report questionnaires that included items relating to demographic variables such as age, sex, education, income, and relationship status, as well as various validated measures exploring perceptions of social interactions following trauma, aspects of trauma disclosure, and PTSD symptoms.

Clinician-Administered PTSD Scale. The Clinician-Administered PTSD ScaleIV (CAPS; Blake et al., 1995) is a semi-structured clinician-administered interview that assesses for a diagnosis of PTSD and symptom severity according to the DSM-IV-TR. In the version of the CAPS used for this study, each of the 17 items corresponding to the DSM-IV symptoms for PTSD were included, as well as items that now correspond to symptoms for the DSM-V diagnostic criteria for PTSD. For each of the items, the clinician rated both the frequency and intensity of the symptom on a scale of 0 to 4 . In order to meet the threshold to be considered clinically significant, a symptom had to be rated with a frequency of at least 1 and an intensity of at least 2. The CAPS also included items that rated social and occupational functioning, global PTSD symptom severity, and the validity of the participant's responses. In the current study, for a diagnosis of PTSD according to the CAPS, a participant had to meet the diagnostic threshold within each of the symptom clusters and have a total symptom severity score of at least 45 .

The original CAPS, using the 17 items corresponding to DSM-IV diagnostic criteria for PTSD, has demonstrated strong psychometric properties across a variety of populations and research settings (Weathers, Keane, \& Davidson, 2001). For example, 
excellent inter-rater reliability has been found for the global severity score $(.89)$ and for total scores of frequency ratings (.92-1.00) and intensity ratings (.93-.98; Hovens et al., 1994). There is also often $100 \%$ inter-rater agreement for a diagnosis of PTSD based on the CAPS (i.e., a Kappa rating of 1.0; Mueser et al., 2001). The CAPS also evidences high internal consistency with Cronbach $\alpha=.85$ to .87 for the three symptom clusters and $\alpha=.97$ for the total score (Blake et al., 1995). The internal reliability of the CAPS used in this study was excellent with Cronbach $\alpha=.94$ for the CAPS total score (herein referred to as CAPS Total).

Social Reactions Questionnaire. The SRQ (Ullman, 2000) was used to assess both positive and negative social reactions that respondents may have received when disclosing their trauma. Respondents were asked how often they received 48 different reactions from persons whom they have told about their trauma using a 5-point Likert scale ranging from 0 (never) to 4 (always). Ullman (2000) examined the psychometric properties of the SRQ with 323 adult survivors of sexual assault. Factor analysis identified seven subscales of the SRQ (Ullman, 2000). Two subscales, Tangible Aid/Information Support (e.g., "helped you get medical care”) and Emotional Support/Validation/Belief (e.g., "told you that you did not do anything wrong") constitute positive types of reactions. Five subscales are considered to represent negative social reactions to disclosure. These include: Victim Blame (e.g., "told you that you could have done more to prevent this experience from occurring"); Treat Differently, referring to treating the victim differently (e.g., "acted as if you were damaged goods or somehow different now"); Distraction/Discouraging, referring to discouraging the victim from talking or thinking about the event (e.g., "told you to stop thinking about it"); Taking 
Control, referring to others' attempts to control the victim's decisions (e.g., "made decisions or did things for you"); and Egocentric Responses (e.g., "has been so upset that he/she needed reassurance from you"). In addition to subscale scores, the mean number of total positive and negative social reactions can be computed. For the purposes of the current study, and given the sample size, the mean number of total positive (herein referred to as SRQ Positive) and negative (herein referred to as SRQ Negative) social reactions were calculated and used in all analyses rather than individual subscales. Ullman (2000) found good convergent validity for the SRQ, as there were significant positive correlations between the SRQ scales with other social support and psychological symptoms measures that theoretically were expected to be associated with the scales (e.g., a correlation of $r=.42$ between positive social reactions and the amount of received support in the past month). Ullman (2000) also found the SRQ to have good test-retest reliability, with correlations ranging from $r=.68$ to .77 . Similarly, good concurrent validity was also demonstrated as the SRQ subscales were correlated with corresponding social reactions coded from open-ended data questions about helpful and unhelpful responses to sexual assault disclosure (Ullman, 2000).

In the current study, internal consistency was excellent for SRQ Positive $(\alpha=.91)$ and SRQ Negative $(\alpha=.94)$ scale scores. One item belonging to the Egocentric Responses scale, "Said he/she felt personally wronged by your experience," was worded incorrectly (worded as "said you felt personally wronged by your experience"). To account for this error, the mean of the other items on the scale was used as a substitute for this item. The item is part of the SRQ Negative scale score. 


\section{Social Acknowledgment as a Victim Questionnaire. The SAQ (Maercker \&}

Mueller, 2004) is a 16-item self-report measure that assesses an individual's perception of society's recognition of them as a survivor or victim and experience of support from family, friends, acquaintances, and local authorities. As such, the construct of social acknowledgment expands on common conceptions of social support that relate to the degree to which an individual experiences emotional and instrumental support from people in their immediate social networks to include how the individual experiences acknowledgment or recognition as a victim in the greater societal context. For example, the SAQ evaluates trauma survivors' perceptions of impersonal experiences such as expressions of opinions in the media regarding the experiences of victims or survivors. This definition takes into account that, in addition to individuals within one's social network, the perception of being judged by social or pressure groups also affects trauma survivors (Maercker \& Mueller, 2004). On the SAQ, participants indicate how much they agree with each of the 16 statements using a 4-point Likert scale ranging from 0 (not at all) to 3 (completely). The SAQ is composed of three subscales: 1) Recognition as a Survivor by Significant Others (herein referred to as SAQ Recognition; e.g., "my friends feel sympathy for what happened to me,"); 2) General Disapproval (herein referred to as SAQ General Disapproval; e.g., "most people cannot understand what I went through,"); and, 3) Friends and Family Disapproval (herein referred to as SAQ Family/Friends; e.g., "my family feels uncomfortable talking about my experiences").

An examination of the psychometrics of the SAQ demonstrated that it has good internal validity. Specifically, as expected, there were moderate negative intercorrelations between the SAQ Recognition subscale with the SAQ General Disapproval and SAQ 
Family/Friends subscales, and moderate positive intercorrelations between SAQ General Disapproval and SAQ Family/Friends subscales. The study also demonstrated that the SAQ has good test-retest reliability over two months with correlations ranging from $r=$ .74 to .85 (Maercker \& Mueller, 2004). In the current study, internal consistency ranged from Cronbach $\alpha=.64$ for SAQ General Disapproval, $\alpha=.72$ for SAQ Recognition and $\alpha=.60$ for SAQ Family/Friends. Although acceptable values of alpha range from .70 to .95 , when there are few items in a scale, low alpha can be expected (Tavakol \& Dennick, 2011). The SAQ General Disapproval and SAQ Family/Friends scales are only composed of five items whereas the SAQ Recognition scale has six items.

Provisions of Social Relations Scale. The Provisions of Social Relations Scale (PSRS; Turner \& Marino, 1995) was used to assess general, nontrauma specific perceived social support. The PSRS is a 22-item scale composed of three subscales: Perceived Partner Support (six items, Cronbach's $\alpha=.88$ ), Perceived Family Support (eight items, Cronbach's $\alpha=.94$ ), and Perceived Friend Support (eight items, Cronbach's $\alpha=$.97). Each subscale assesses the degree to which the respondent feels close to the target support provider (i.e., romantic partner, family, and friend), perceives the support provider is willing to take the time to talk and have communication of worth, has confidence that the support source will be there when needed, and the belief that the support source has confidence in the respondent. Respondents rate their agreement with each item using a 4-point Likert scale ranging from 1 (Not at all like my Experience) to 4 (Very much like my Experience).

All participants were administered the Perceived Family Support and Perceived Friend Support scales. Respondents who did not have a romantic partner did not complete 
the Perceived Partner Support scale. A total score was calculated using the mean of the appropriate scales (Turner \& Marino, 1995). In the current study, internal consistency for the PSRS total score (herein referred to as PSRS Total) was excellent, with Cronbach $\alpha=$ .97 for the total score calculated with all three scales and Cronbach $\alpha=.96$ for the total score calculated without the Perceived Partner scale for those without romantic partners.

Disclosure of Trauma Questionnaire. The DTQ (Mueller et al., 2000) is a 34item self-report tool that measures aspects of how a person communicates about their traumatic event including attitudes and intentions to disclose traumatic events. The DTQ is composed of three subscales: 1) Urge to Talk, which assesses respondents' urge to talk or attempts to express themselves with regard to their experiences (e.g., "it is important for me to repeatedly talk about what happened and how it happened"); 2) Reluctance to Talk, which assesses resistance to disclose the traumatic event to others (e.g., "I find it difficult to talk to people about the incident"); and 3) Emotional Reactions, which assesses emotional reactions while disclosing (e.g., "describing the event makes me feel very sad"). The DTQ has been found to have good psychometric properties with Cronbach's $\alpha$ 's $=0.72$ to 0.82 for the three subscales (Fankhauser et al., 2011) and testretest reliability ranging between $r=.076$ to 0.89 (Mueller et al., 2000). Given the small sample size in the current study, in order to decrease the chance of Type I error and increase power, only the total score of the DTQ (herein referred to as DTQ Total) was entered into a regression analysis, rather than three separate analyses to test for interactions between each of the three subscales and social interaction measures. In the current study, internal consistency for the DTQ Total was excellent with Cronbach $\alpha=$ .91 . 
MINI International Neuropsychiatric Interview. The MINI International Neuropsychiatric Interview (MINI; Lecrubier et al., 1997) is a validated structured diagnostic interview that assesses for past and current DSM-IV-TR Axis I disorders. The MINI has good concordance with other clinician-administered tools that detect for DSM disorders (Sheehan et al., 1998). The MINI was used to describe and characterize the mental health conditions of the current sample.

\section{Procedure}

Individuals interested in participating in the study contacted the research personnel by telephone or email according to the contact information provided in the advertisements. Once initial contact was made with the research personnel, interested participants were given more information about the study. Verbal informed consent was obtained over the telephone to participate in the study. Consent was obtained to keep the information gathered from the telephone screen regardless of whether the individual qualified for the study. This was done in order to determine the characteristics of excluded individuals. After eligibility was established, participants meeting inclusion criteria were sent informed consent forms by mail or were directed to a link to view the informed consent forms online. The research personnel also contacted participants by telephone or met with them in person to review specific elements of the informed consent form. Participants were given the option of signing informed consent forms in person, electronically via the Internet, or by returning them via a stamped return envelope.

The full procedure for the parent study entailed four assessments over the course of 12 months, with participants completing assessments every 3 months. For the purposes of the current research, only data from the Time 1, initial assessment, were used. A 
graduated remuneration system was used to compensate participants for taking the time to complete the study. Specifically, each participant was paid $\$ 60$ for the first assessment, $\$ 70$ for the second assessment, $\$ 80$ for the third assessment, and $\$ 90$ for the final assessment, resulting in a total of $\$ 300$ per individual for completing all assessment points.

\section{Data Analysis}

SPSS version 19.0 (IBM Corp, 2010) was used to analyze study data and the macro PROCESS for SPSS 19.0 (Hayes, 2012) was used to test for moderation in Hypothesis 2. In order to account for missing data on relevant measures, missing items were replaced with the mean of the other subscale items when $75 \%$ of the items on a subscale were completed.

The PROCESS macro was used to test the moderation models. These models specify linear interactions between the predictor variable (i.e., negative social interactions) and each of the potential moderators (i.e., sex, trauma type, and disclosure style) using an ordinary least squares framework. PROCESS also provides the option to mean center all predictor variables used to form the products when estimating the moderated path, an option that ensures that the coefficients for the two variables that define the interaction product (i.e., negative social interactions and the demographic or disclosure variables) will be interpretable within the range of data. PROCESS tests whether the coefficient for the product (interaction term) is significantly different from zero and provides the unique proportion of the total variance in the outcome that can be accounted for by the interaction term. PROCESS also uses bootstrapping methods, a nonparametric procedure that does not require the sampling distribution to be normally 
distributed. Bootstrapping entails repeatedly sampling from the data set and estimating the indirect effects in each resampled data set. This process is repeated thousands of times to yield an empirical estimate of the sampling distribution that is used to construct confidence intervals for the interaction effect. When there is a significant interaction and the moderator is dichotomous, PROCESS allows for interpretation of the conditional effects of the predictor (simple slopes) at each of the two values of the moderator, along with a standard error, $t$, and $p$ value. When the interaction is significant and the moderator is a continuous variable, the conditional effects of the predictor variable are estimated when the moderator is equal to the mean, as well as plus and minus one standard deviation from the mean. PROCESS also allows researchers to select any value of the moderator at which to estimate the conditional effect of the predictor on the outcome.

Hypothesis 1. Negative social interactions will predict PTSD symptoms above and beyond measures of general social support and positive social interactions. Specifically, SRQ Negative and SAQ General Disapproval scales will emerge as the only significant predictors of PTSD symptoms when considered simultaneously in a regression analysis with measures of positive social interactions and general social support. In order to test this hypothesis, first, bivariate correlations between overall PTSD symptom severity (as measured by the CAPS Total) and PSRS Total, SRQ Negative, SRQ Positive and each of the three SAQ subscales were conducted.

Social factors found to have significant bivariate correlations with PTSD symptom severity were entered into a regression model to simultaneously predict clinician-rated PTSD symptoms. Specifically, CAPS Total scores were entered as the dependent variable and the social interaction measures that had significant bivariate 
correlations with the CAPS were entered as the independent variables. A power analysis determined that, if all six social interaction scales are entered into a multiple regression model, small effect sizes $\left(F^{2}=0.15\right)$, could be detected at $\alpha=.05$, two tailed, and at a power of greater than 99 .

Hypothesis 2. The relationship between negative social interactions and PTSD symptoms will be moderated by sex, trauma type, and disclosure style as measured by DTQ Total scores such that negative social interactions will be relatively more predictive of PTSD symptoms for females, survivors of sexual assault and those with dysfunctional disclosure styles. Separate moderation models were built using stepwise regression methods in PROCESS. These models also controlled for the potential roles of participants' education (highest level of education achieved), ethnicity, and age, because these variables have been found to be associated with PTSD symptoms (Brewin et al., 2000). In each of the models, interaction terms were built between the social interaction scales found to be significant predictors of PTSD symptoms in Hypothesis 1 and the potential moderators of sex, dysfunctional disclosure style, and trauma type while controlling for the covariates of education, age, and ethnicity. More specifically, the social interaction scales found to be significant predictors of PTSD symptoms in the model were entered into interaction models with sex, DTQ, and trauma type with age, ethnicity, and education entered into the model as covariates. Given the sample size and that multiple levels of ethnicity were captured in the data, dummy coding was used with the ethnicity variable to limit the variable to two levels. Caucasian was used as the reference variable, indicated by " 0 " and other ethnicities indicated with " 1 " as half of the sample identified as Caucasian. For each analysis, the number of bootstraps was set at 
5,000 with confidence intervals of $95 \%$. If the confidence intervals of the interaction effect did not include a 0 , then the null hypothesis of no significant interaction was rejected. Given that multiple analyses were conducted, Bonferroni corrections were used to control for Type I error for the overall models such that the $p$ values were divided by the total number of models in the study. A power analysis determined that if three covariates, one social interaction scale, and one moderator variable (i.e., sex, dysfunctional disclosure style, trauma type) were entered into a simple moderation model, an interaction with a moderate to large effect size, (cumulative $R^{2}$ of 0.25 ) could be detected at $\alpha=0.05$, two tailed, and at a power of greater than .99 .

Model(s) for sex as a moderator. Negative social interactions will more strongly predict PTSD symptoms for females compared with males. To test this hypothesis, simple moderation modeling (PROCESS model 1) in PROCESS was used with sex treated as a dichotomous variable. With dichotomous variables such as sex, PROCESS produces simple slopes that allow for interpretation of the conditional effect of the significant moderator on the predictor at each level of the moderator (i.e., female and male). The standard error, $t$ and $p$ value are also provided. For each social interaction scale found to be significantly associated with PTSD in Hypothesis 1, a unique model was built with this social interaction scale and sex as a moderator to predict PTSD symptoms.

Model(s) for disclosure style as a moderator. Negative social interactions will predict PTSD symptoms for those with highly dysfunctional disclosure styles. To test this hypothesis, the DTQ Total score was entered into a simple moderation model (PROCESS model 1) in PROCESS. With continuous moderator variables, the conditional effects of 
the predictor (i.e., negative social interactions) are typically estimated using values of the moderator that are derived from the mean. The mean, one standard deviation above the mean, and one standard deviation below the mean are typically used to indicate moderate, relatively high, and relatively low values of the moderator, respectively. Therefore, the mean, one standard deviation below the mean, and one standard deviation above the mean will be used to delineate levels of dysfunctional disclosure style. One standard deviation above the mean will represent high levels of dysfunctional disclosure. However, PROCESS also provides the option to examine the conditional effect of the predictor on the outcome at any value of the moderator and this option may be used depending on the findings from the current study. A model was built for each social interaction measure found to be a significant predictor of PTSD symptoms in Hypothesis 1 to test for an interaction between disclosure style and that social interaction measure on PTSD symptoms.

Model(s) for trauma type as a moderator. Negative social interactions and PTSD symptoms will be moderated by trauma type, such that negative social interactions will be relatively more predictive of PTSD symptoms for survivors of sexual trauma versus other types of trauma. In order to test this hypothesis, PROCESS model 2 was used. This model is used because trauma type is a multi-categorical variable.

One of the drawbacks of PROCESS is that it is not designed to properly model multicategorical variables. However, Hayes (2015) describes a "hacking” process whereby variables with three categories can be modelled using PROCESS model 2 to estimate a simple moderation model (PROCESS model 1). As this "hacking” process only allows for a maximum of three categories for multicategorical variables, the types of 
trauma that led participants to study enrolment were grouped into one of three categories: sexual interpersonal violence $(n=30)$, nonsexual interpersonal violence $(n=46)$, and other traumas $(n=73)$.

PROCESS model 2 is typically used when two moderator variables are entered into the same equation but with a multicategorical variable with three levels, such as trauma type, dummy coding can be used to treat the different levels of the categorical variable as if they are separate moderators, each with its own interaction term with the predictor (Hayes, 2015). Two of the levels are coded with " $1 \mathrm{~s}$ " and "0s" to indicate their category, and the third category is coded as " 0 " " 0 ." Using this coding system, when interpreting the data, the two interaction terms are considered to represent one moderator variable. For the current study, sexual interpersonal violence was coded as the comparison condition (“0”, “0”). With PROCESS, if there is an interaction effect found, plots can be generated to interpret conditional effects. PROCESS also allows for two pairwise comparisons between the conditional effects (Hayes, 2015). 


\section{Results}

See Table 1 for participant demographics and Table 2 for current and lifetime rates of mental health conditions in the current sample. The majority of participants were females (69.6\%) and identified as Caucasian (49.7\%). Just over half of the participants were single or never married (52.7\%), and roughly, one third had children $(36.1 \%)$. The majority reported their highest level of education as a college diploma (30.9\%) or an undergraduate degree (37.6\%). Participants ranged in age from 18 to 70 years old $(M=37.67, S D=13.95)$ and nearly one half $(46.3 \%)$ were employed at the time of the assessment. Almost one third of the current sample met diagnostic criteria for a current mood disorder and nearly $20 \%$ met criteria for a current anxiety disorder. In terms of the traumatic event that led to study enrollment, $30.1 \%$ were exposed to accidents $(n=46)$, $26.2 \%$ to noncombat physical assault $(n=39), 20.1 \%$ to sexual assault $(n=30), 8.7 \%$ to sudden death $(n=13), 1.3 \%$ to illness $(n=3), 1.3 \%$ to threats by another person $(n=2)$, $3.4 \%$ to robbery or home invasion $(n=5), 6.7 \%$ to another trauma $(n=10)$ and $0.7 \%$ were held hostage $(n=1)$. The majority of participants directly experienced the traumatic event $(78.5 \%, n=117), 18.1 \%$ witnessed the traumatic event $(n=27), 2.0 \%$ learned about the traumatic event $(n=3)$, and $1.3 \%$ reported being exposed to the traumatic event by another means $(n=2)$. Based on the CAPS, $65(43.6 \%)$ participants met criteria for a DSM-IV-TR diagnosis of PTSD. The mean score for the sample on the CAPS $(M=$ 46.53, $S D=29.97)$ was consistent with moderate PTSD symptom severity, with scores ranging from 0 (considered asymptomatic) to 112 (considered extreme PTSD symptomatology). Means and standard deviations for scale scores are presented in Table 1. 
Table 1

Demographics of the Sample

Variable

$\mathrm{N}(\%)$

Female

103 (69.6)

Employed at time of assessment

68 (46.3)

Relationship status

Single/never married

$78(52.7)$

In a committed relationship/never married

22 (14.9)

Married

19 (12.8)

Separated/Divorced/Widowed

29 (19.6)

Have children

$53(36.1)$

Highest level of education

High school diploma

$32(21.5)$

College diploma

46 (30.9)

Undergraduate degree

56 (37.6)

Masters degree

$10(6.7)$

Doctoral degree

$1(0.7)$

Ethnicity

Aboriginal

7 (4.8)

Arab/West Asian (e.g., Armenian, Egyptian)

4 (2.7)

Black (e.g., African, Haitian, Jamaican)

$20(13.6)$

East Asian (e.g., Chinese, Japanese)

$9(6.1)$

Latin American

4 (2.7)

South Asian

$10(6.8)$

South East Asian

$6(4.1)$

White (Caucasian)

74 (49.7)

Mixed

$4(2.7)$

Other

$9(6.0)$ 
Age (years)

37.67 (13.95)

18-70

CAPS Total

46.53 (29.97) 0-112

SAQ General Disapproval

$6.24(3.05)$

0-14

SAQ Recognition

$7.44(3.91)$

$0-18$

SAQ Family/Friends

$6.58(3.31)$

0-15

SRQ Positive

$2.99(.78)$

$1-4.6$

SRQ Negative

$1.81(.71)$

$.08-3.72$

PSRS Total

$20.59(6.24)$

7.33-32

DTQ Total

$67.63(26.56)$

$12-134$

Notes . CAPS Total $=$ Clinician-Administered Posttraumatic Stress Disorder Scale total symptom severity score; SAQ General Disapproval = Social Acknowledgment as a Victim Questionnaire General Disapproval subscale score; SAQ Recognition = Social Acknowledgment as a Victim Questionnaire Recognition subscale score; SAQ

Family/Friends $=$ Social Acknowledgment as a Victim Questionnaire Family and Friends disapproval subscale score; SRQ Negative = Social Reactions Questionnaire mean of negative interaction scale scores; SRQ Positive $=$ Social Reactions Questionnaire mean of positive interaction scale scores; PSRS Total = Provisions of Social Relations Scale total scale score; DTQ Total $=$ Disclosure of Trauma Questionnaire total scale scores . Variation in sample size across variables is due to missing data. 
Table 2

Prevalence of Diagnostic and Statistical Manual of Mental Disorders, Fourth Edition, Text Revision Current and Lifetime Mental Health Conditions among Participants

\begin{tabular}{lccc}
\hline & $\begin{array}{c}\text { Current } \\
\end{array}$ & & $\begin{array}{c}\text { Lifetime } \\
\text { Mood Disorder }\end{array}$ \\
Anxiety Disorder (excluding PTSD) & $26(28.9 \%)$ & & $57(38.3 \%)$ \\
PTSD (related to another event) & $15(10.1 \%)$ & & $19(12.8 \%)$ \\
Substance Use Disorder & $26(17.5 \%)$ & \\
Psychotic Disorder & $2(1.3 \%)$ & $3(2.0 \%)$ \\
Eating Disorder & $5(3.4 \%)$ & \\
\hline
\end{tabular}

Note. PTSD prevalence rates reflect PTSD resulting from a traumatic event other than the event leading to participation in the current study. 


\section{Tests of Normality of Data}

To test for normality, $z$-tests for skewness and kurtosis were calculated for each measure. PTSD symptoms, as measured by CAPS Total scores, had a skew of .22 (SE = $.20)$ and kurtosis of $-.52(S E=.40)$. SAQ General Disapproval had a skew of $.08(S E=$ $.20)$ and kurtosis of $-.48(S E=.40)$, SAQ Recognition had a skew of .42 $(S E=.20)$ and kurtosis of .01 $(S E=.40)$, SAQ Family/Friends had a skew of $.03(S E=.20)$ and kurtosis of -.57 $(S E=.40)$, SRQ Negative had a skew of $.25(S E=.20)$ and kurtosis of -.04 $(S E=$ $.41)$, and PSRS Total had a skew of -.07 $(S E=.20)$ and kurtosis of -.52 $(S E=.40)$. The SRQ Positive had a skew of $-.50(S E=.20)$ and kurtosis of $-.15(S E=.40)$, the DTQ had a skew of -.15 $(S E=.20)$ and a kurtosis of $-.44(S E=.40)$, and age had a skew of .38 (SE $=.20)$ and kurtosis of $-.93(S E=.40)$. In medium sample sizes ranging between $N=50$ to $N=300$, absolute $z$ values of greater than 3.29 are considered to represent significant skewness or kurtosis (Kim, 2013); thus none of the data were transformed.

\section{Hypothesis 1}

Bivariate correlations with CAPS. As predicted, all the social interaction scale scores, except for scores on the SRQ Positive scale, had significant bivariate correlations with CAPS Total scores (see Table 3). Similarly, scales measuring negative social interactions had significant positive correlations with CAPS Total scores. Specifically, the SAQ General Disapproval scale had a significant moderate positive correlation with the CAPS Total scores, as did the SAQ Family/Friends scale, and SRQ Negative scale. PSRS Total scores had a weak significant negative correlation with CAPS scores, as did the SAQ Recognition (measure of positive social interaction) scale with CAPS Total scores. SRQ Positive scores were not significantly correlated with CAPS Total scor 
Table 3

Correlations between Social Interaction Scales, Disclosure Style, Age, Education, and Clinician-Rated Posttraumatic Stress Disorder Symptom Severity

\begin{tabular}{|c|c|c|c|c|c|c|c|c|c|c|}
\hline & 1 & 2 & 3 & 4 & 5 & 6 & 7 & 8 & 9 & 10 \\
\hline 1. CAPS Total & - & $.57 * * *$ & $-.29 * * *$ & $.49 * * *$ & $.35^{* * *}$ & -.05 & $-.30 * * *$ & $.53 * * *$ & .16 & -.04 \\
\hline 2. SAQ General Disapproval & & - & $-.22 * *$ & $.47 * * *$ & $.35 * * *$ & -.03 & $-.30 * * *$ & $.59 * * *$ & .03 & -.07 \\
\hline 3. SAQ Recognition & & & - & $-.35^{* *}$ & -.06 & $.44 * * *$ & $.29 * *$ & .07 & .14 & $.20 *$ \\
\hline 4. SAQ Family/Friends & & & & - & $.24 * *$ & $-.26 * *$ & $-.29 * *$ & $.33 * * *$ & -.04 & .02 \\
\hline 5. SRQ Negative & & & & & - & .15 & $-.24 * *$ & $.40 * * *$ & .13 & .02 \\
\hline 6. SRQ Positive & & & & & & - & $.24 * *$ & $.17 *$ & -.02 & .12 \\
\hline 7. PSRS Total & & & & & & & - & $-.25 * *$ & -.14 & .12 \\
\hline 8. DTQ Total & & & & & & & & - & .12 & $.17 *$ \\
\hline 9. Age & & & & & & & & & - & .05 \\
\hline 10. Education & & & & & & & & & & - \\
\hline
\end{tabular}

Notes. CAPS Total $=$ Clinician-Administered Posttraumatic Stress Disorder Scale total symptom severity score; SAQ General Disapproval $=$ Social Acknowledgment as a Victim Questionnaire General Disapproval subscale score; SAQ Recognition = Social Acknowledgment as a Victim Questionnaire Recognition subscale score; SAQ Family/Friends = Social Acknowledgment as a Victim Questionnaire Family and Friends disapproval subscale score; SRQ Negative = Social Reactions Questionnaire mean of negative 
interaction scale scores; SRQ Positive $=$ Social Reactions Questionnaire mean of positive interaction scale scores; PSRS Total $=$ Provisions of Social Relations Scale total scale score; DTQ Total = Disclosure of Trauma Questionnaire total scale scores; Education $=$ highest level of education.

$$
\begin{gathered}
* * * p<.001 \\
* * p<.01 \\
* p<.05
\end{gathered}
$$


Regression analysis. The five social scales that significantly correlated with CAPS Total scores (i.e., SRQ Negative, SAQ General Disapproval, SAQ Family/Friends, SAQ Recognition and PSRS Total) were simultaneously entered into a linear regression to predict CAPS Total scores. The model accounted for a significant proportion of the variance in CAPS Total scores. Among the predictors entered into the model, the SAQ General Disapproval scale, and SAQ Family/Friends scales, emerged as significant predictors of PTSD symptoms, but the SAQ Recognition, SRQ Negative, and PSRS Total scales were not significant (see Table 4). 
Table 4

Multiple Regression Analyses Predicting Clinician-Rated Posttraumatic Stress Disorder Symptom Severity from Negative, Positive, and General Measures of Social Interactions

\begin{tabular}{lcccc}
\hline Predictor & $B(\mathrm{SE} B)$ & $\beta$ & $t$ & $p$ \\
\hline SAQ General Disapproval & $3.24(.69)$ & .37 & 4.70 & $<.001$ \\
SAQ Recognition & $-.89(.51)$ & -.13 & -1.75 & .08 \\
SAQ Family/Friends & $1.97(.66)$ & .23 & 2.99 & .003 \\
PSRS Total & $-.24(.32)$ & -.05 & -.74 & .46 \\
SRQ Negative & $5.32(2.79)$ & .14 & 1.91 & .06 \\
\hline
\end{tabular}

Notes. $\mathrm{CI}=$ confidence interval; SAQ General Disapproval = Social Acknowledgment as a Victim Questionnaire General Disapproval subscale score; SAQ Recognition = Social Acknowledgment as a Victim Questionnaire Recognition subscale score; SAQ Family/Friends = Social Acknowledgment as a Victim Questionnaire Family and Friends disapproval subscale score; PSRS Total = Provisions of Social Relations Scale total scale score; SRQ Negative = Social Reactions Questionnaire negative interaction mean scores. The model accounted for a significant proportion of the variance in CAPS scores, $R^{2}=.43, F(5,132)=19.85, p<.001$. 


\section{Hypothesis 2}

Six models were built to test for significant interactions between each social interaction scale found to be a unique significant predictor of CAPS Total scores (i.e., SAQ General Disapproval, SAQ Family/Friends) and each of the three hypothesized moderators (i.e., sex, trauma type, disclosure style). For each of the three hypothesized moderators, separate models were built with each SAQ scale entered as the independent variable and CAPS Total scores entered as the dependent variable. Participants' education (highest level of education achieved), ethnicity (with Caucasian and non Caucasian ethnicity, Caucasian used as the reference group), and age were entered into each model as covariates.

Models with sex as a moderator. In each of the two models to test whether sex moderated the relationship between social interactions and PTSD symptom severity, neither interaction term was a significant predictor of CAPS Total scores. In both models, age was a significant covariate predicting PTSD severity (see Tables 5 and 6). 
Table 5

Sex as a Moderator of the Effect of General Societal Disapproval on Clinician-Rated Posttraumatic Stress Disorder Symptom Severity

\begin{tabular}{|c|c|c|c|c|}
\hline Predictor & $b$ & $S E(\mathrm{~B})$ & $t$ & $p$ \\
\hline Sex (centered) & 3.41 & 8.74 & .39 & .70 \\
\hline SAQ General Disapproval (centered) & 7.12 & 1.83 & 3.89 & $<.001$ \\
\hline Sex X SAQ General Disapproval & -1.55 & 1.23 & -1.26 & .21 \\
\hline Age & .30 & .14 & 2.22 & .03 \\
\hline Non Caucasian Ethnicity & -2.65 & 3.72 & -.72 & .48 \\
\hline Education & .00 & .01 & .03 & .98 \\
\hline
\end{tabular}

Notes. SAQ General Disapproval = Social Acknowledgment as a Victim Questionnaire General Disapproval subscale score; Non Caucasian Ethnicity = ethnicity reported as non Caucasian; Education $=$ highest level of education. $R^{2}=.36$ 
Table 6

Sex as a Moderator of the Effect of Family and Friends Disapproval on Clinician-Rated Posttraumatic Stress Disorder Symptom Severity

\begin{tabular}{lcccc}
\hline Predictor & $b$ & $S E(\mathrm{~B})$ & $t$ & $p$ \\
\hline Sex (centered) & -1.49 & 11.07 & -.14 & .89 \\
SAQ Family/Friends (centered) & 4.49 & 1.95 & 2.30 & .03 \\
Sex X SAQ Family/Friends & -.36 & 1.56 & -.23 & .82 \\
Age & .39 & .15 & 2.64 & .01 \\
Non Caucasian Ethnicity & -3.55 & 4.06 & -.87 & .38 \\
Education & -.01 & .01 & -.68 & .50 \\
\end{tabular}

Notes. SAQ Family/Friends = Social Acknowledgment as a Victim Questionnaire Family and Friends Disapproval subscale score; Non Caucasian Ethnicity = ethnicity reported as non Caucasian; Education $=$ highest level of education. $R^{2}=.28$ 
Models with disclosure style as a moderator. Neither of the interaction terms in the two models to test the hypothesis that negative social interactions would significantly predict PTSD symptoms for those with more dysfunctional disclosure styles was statistically significant. None of the covariates were significant in the model exploring the interaction between DTQ Total scores and SAQ General Disapproval. However, in the model exploring the interaction between DTQ Total scores and SAQ Family/Friends, age, ethnicity, and education were found to be marginally statistically significant predictors of CAPS Total scores (see Tables 7 and 8). 


\section{Table 7}

Dysfunctional Disclosure Style as a Moderator of the Effect of General Societal Disapproval on Clinician-Rated Posttraumatic Stress Disorder Symptom Severity

\begin{tabular}{|c|c|c|c|c|}
\hline Predictor & $b$ & $S E(\mathrm{~B})$ & $t$ & $p$ \\
\hline DTQ Total (centered) & .28 & .17 & 1.64 & .10 \\
\hline SAQ General Disapproval (centered) & 2.42 & 1.84 & 1.31 & .19 \\
\hline DTQ Total x SAQ General Disapproval & .01 & .03 & .43 & 67 \\
\hline Age & .19 & .13 & 1.47 & .15 \\
\hline Non Caucasian Ethnicity & -5.68 & 3.68 & -1.54 & .13 \\
\hline Education & -.01 & .01 & -.95 & .34 \\
\hline
\end{tabular}

Notes. DTQ Total = Disclosure of Trauma Questionnaire total scale scores; SAQ General Disapproval = Social Acknowledgment as a Victim Questionnaire General Disapproval subscale score; Non Caucasian Ethnicity = ethnicity reported as non Caucasian; Education $=$ highest level of education. $R^{2}=.41$ 
Table 8

Dysfunctional Disclosure Style as a Moderator of the Effect of Family and Friends Disapproval on Clinician-Rated Posttraumatic Stress Disorder Symptom Severity

\begin{tabular}{|c|c|c|c|c|}
\hline Predictor & $b$ & $S E(\mathrm{~B})$ & $t$ & $p$ \\
\hline DTQ Total (centered) & .23 & .16 & 1.42 & .16 \\
\hline SAQ Family/Friends (centered) & .43 & 1.79 & .24 & .82 \\
\hline DTQ Total x SAQ Family/Friends & .04 & .02 & 1.50 & .14 \\
\hline Age & .24 & .13 & 1.79 & .08 \\
\hline Non Caucasian Ethnicity & -6.85 & 3.63 & -1.89 & .06 \\
\hline Education & -.03 & .01 & -1.88 & .06 \\
\hline
\end{tabular}

Notes. DTQ Total $=$ Disclosure of Trauma Questionnaire total scale scores; SAQ Family $/$ Friends $=$ Social Acknowledgment as a Victim Questionnaire Family and Friends Disapproval subscale score; Non Caucasian Ethnicity = ethnicity reported as non Caucasian.

Education = highest level of education. $R^{2}=.45$ 
Models with trauma type as a moderator. Two models were built to test the hypothesis that negative social interactions would be stronger predictors of PTSD symptoms for survivors of sexual interpersonal violence versus nonsexual interpersonal violence, or other types of traumas. Dummy coding was used, with sexual interpersonal violence treated as the reference variable. Neither interaction term in the models was a significant predictor of CAPS Total scores. In these models, age emerged as a significant covariate predicting PTSD symptoms (see Tables 9 and 10). 
Table 9

Trauma Type as a Moderator of the Effect of General Societal Disapproval on Clinician-Rated Posttraumatic Stress Disorder Symptom Severity

\begin{tabular}{lcccc}
\hline Predictor & $b$ & $S E(\mathrm{~B})$ & $t$ & \multicolumn{1}{c}{. } \\
\hline Trauma type nonsexual (centered) & & 13.83 & .53 & .60 \\
SAQ General Disapproval (centered) & 7.31 & 1.50 & 3.49 & $<.001$ \\
Trauma type nonsexual x SAQ General Disapproval & -1.60 & 1.81 & -.88 & .38 \\
Trauma type other & -10.90 & 12.72 & -.86 & .39 \\
Trauma type other x SAQ General Disapproval & -.80 & 1.72 & -.46 & .64 \\
Age & .35 & .13 & 2.71 & .01 \\
Non Caucasian Ethnicity & -1.94 & 3.60 & -.54 & .59 \\
Education & -.00 & .01 & -.13 & .89 \\
\end{tabular}

Notes. Trauma type nonsexual = nonsexual interpersonal violence; SAQ General Disapproval = Social Acknowledgment as a Victim Questionnaire General Disapproval subscale score; Trauma type other = trauma type, other type of trauma; Non Caucasian Ethnicity = ethnicity reported as non Caucasian; Education $=$ highest level of education. $R^{2}$ due to interaction $=.004$ 
Table 10

Trauma Type as a Moderator of the Effect of Family and Friends Disapproval on Clinician-Rated Posttraumatic Stress Disorder Symptom Severity

\begin{tabular}{|c|c|c|c|c|}
\hline Predictor & $b$ & $S E(\mathrm{~B})$ & $t$ & $p$ \\
\hline Trauma type nonsexual (centered) & -.2 .20 & 13.94 & -.16 & .88 \\
\hline SAQ Family/Friends (centered) & 3.05 & 1.26 & 2.43 & .02 \\
\hline Trauma type nonsexual x SAQ Family/Friends & .08 & 1.69 & .05 & .96 \\
\hline Trauma type other & -23.25 & 12.51 & -1.86 & .07 \\
\hline Trauma type other x SAQ Family/Friends & .98 & 1.57 & .63 & .53 \\
\hline Age & .46 & .14 & 3.24 & .001 \\
\hline Ethnicity & -3.03 & 4.02 & -.75 & .45 \\
\hline Education & -.01 & .01 & -.84 & .40 \\
\hline
\end{tabular}

Notes. Trauma type nonsexual $=$ nonsexual interpersonal violence; SAQ Family/Friends = Social Acknowledgment as a Victim Questionnaire Family and Friends disapproval subscale score; Non Caucasian Ethnicity = ethnicity reported as non Caucasian; Trauma type other $=$ trauma type, other type of trauma; Education $=$ highest level of education. $\Delta R^{2}$ due to interaction $=.00$ 


\section{Discussion}

The purpose of the current study was to extend the literature by examining the aspects of social interactions most strongly associated with PTSD symptoms in recently traumatized individuals and the factors that moderate these relationships. Consistent with Hypothesis 1, negative aspects of posttraumatic social interactions, specifically general societal disapproval and disapproval from family and friends were found to be associated with PTSD symptoms, whereas positive posttraumatic social interactions were not significantly associated with PTSD symptoms. However, contrary to Hypothesis 2, the relationships between general societal disapproval and family and friends disapproval and PTSD symptoms did not vary based on participant's sex, trauma disclosure style, or trauma type. Taken together, these findings suggest that negative social interactions are more strongly associated with PTSD severity than positive interactions. Positive interactions may not be as integral to trauma recovery at 6 months following trauma exposure. Negative interactions seem to be important in terms of trauma recovery regardless of survivors' sex, type of trauma experienced, and style of disclosing about their trauma.

\section{Negative Versus Positive Social Interactions: Aim 1}

This study aimed to examine whether negative social interactions were more strongly associated with PTSD symptom severity than positive ones. Three positive facets of social interaction were explored, including general social support (measured by the PSRS) and, trauma-specific support, such as positive social reactions to trauma disclosure (as measured by the SRQ Positive) and recognition of one's experience as a survivor of trauma (as measured by the SAQ Recognition). Three measures of negative 
social interactions following trauma were also included. These were general societal disapproval (as measured by SAQ General Disapproval scores), disapproval from family and friends (as measured by SAQ Family/Friends scores) and negative reactions to trauma disclosure (as measured by the SRQ Negative).

Overall, mean values on measures of social interactions were comparable to those found in other studies. For example, means on the SRQ Negative and Positive scales were similar to those found in samples of ethnically diverse females exposed to sexual assaults (Ullman et al., 2006; Ullman et al., 2007) and samples of college students who survived rape (roughly half of the sample had experienced the trauma within 2 years of the study; Littleton et al., 2010). On average, scores on the SAQ indicated low societal acknowledgment and were comparable to those found in German crime victims assessed at 5 months posttrauma who also exhibited high rates of PTSD symptoms (Mueller et al., 2008). SAQ scores in the current study indicated slightly lower acknowledgment as a victim than those found in studies of developmental aid workers and former political prisoners wherein participants exhibited lower rates of PTSD (Jones et al., 2006; Maercker \& Mueller, 2004). Perhaps aid workers and political prisoners endorse greater societal appreciation and recognition, given the nature of their traumas (these traumas may potentially be associated with less societal stigma than events involving interpersonal violence; Maercker et al., 2009).

As hypothesized, bivariate correlations revealed that negative social interactions were positively correlated with PTSD symptom severity and positive interactions were negatively correlated with PTSD symptom severity. This suggests that negative interactions are related to greater PTSD symptom severity whereas positive ones to less 
severe symptom severity. Interestingly, and in line with past research (e.g., Ullman \& Filipas, 2001; Ullman et al., 2007), the SRQ Positive scale, a measure of positive social reactions to trauma disclosure, was the only scale not significantly correlated with PTSD severity. When considered simultaneously in a multiple linear regression analysis, the SAQ Family/Friends and SAQ General Disapproval scales were the only significant predictors of clinician-rated PTSD symptoms, although SRQ Negative scores were marginally statistically significant in the regression. This finding is consistent with past cross-sectional and longitudinal research using the SRQ in samples of sexual assault survivors (e.g., Ullman \& Filipas, 2001; Ullman et al., 2007). For example, past studies exploring negative and positive social interactions among survivors of diverse traumatic events (e.g., assault, accidents) also found that negative interactions were more strongly associated with PTSD symptoms. These findings suggest that negative social interactions are more related to trauma recovery than positive interactions.

The current results provide support for the social negativity hypothesis (Major et al., 1997) that holds that negative interactions may have a stronger influence on mental health outcomes because people tend to give more weight to negative information than positive. As such, the detrimental effects of negative interactions may outweigh the protective influence of positive social experiences. One explanation for the finding that negative interactions were more strongly related to PTSD severity than positive ones may be that individuals with PTSD have a bias to recall more negative than positive interactions. The measures used in this study are of perceptions of social support and it is possible that symptoms of PTSD or comorbid mental health problems (e.g., depression, insomnia; Barber \& Budnick, 2015; Gilboa; Schechteman, Erhard-Weiss \& Jeczmien, 
2002) increase reporting of negative interactions on these measures. The construct of perceived social interactions is considered, in part, a measure of bias in evaluation of and memory for supportive behaviours (Lakey \& Cassady, 1990). For instance, some actions taken by support providers in an effort to assist a victim could be coded on a measure of received social interactions as positive, but interpreted negatively by a trauma survivor as reinforcing their lack of control. Individuals with higher PTSD severity may be more likely to report a greater number of negative social interactions than positives ones. Interestingly, in the current study means from the positive and negative interaction scales were comparable. This again suggests that although participants' were as likely to report positive social interactions as negative ones, positive interactions are relatively less important in terms of PTSD severity.

The two scales found to be most strongly predictive of PTSD symptoms (SAQ General Disapproval and SAQ Family/Friends) capture a trauma survivor's perception of general lack of acknowledgment of one's experience as a victim from both one's wider social network and closer network of family and friends. Past studies exploring the SAQ in relation to trauma recovery have also found the SAQ General Disapproval and SAQ Family/Friends scales to be more strongly associated with PTSD than the SAQ Recognition scale (Maercker et al., 2009; Mueller et al., 2008; Wagner et al., 2012). This may be understood by considering the symptoms of PTSD, particularly symptoms such as negative alterations in cognitions and moods, that include negative beliefs or expecations about onself, others, or the world, as well as feelings of detachment or estrangment from others (American Psychiatric Association, 2013). Individuals may develop these symptoms from perceptions of a lack of acknowledgment of their 
experience as a trauma survivor from both family, friends, and society in general, rendering general feelings of detachment or negative expectations of the future and others. Avoidance symptoms, such as efforts to avoid conversations about the traumatic event or activities, people or places that serve as reminders of the event may be perpetuated by a general feeling of disapproval from society or friends or family. When one feels as though others do not acknowledge their experience as a victim, they may feel less comfortable in situations that remind them of the trauma.

The SRQ Negative scale, a measure of negative reactions to trauma disclosure, did not emerge as a unique predictor of PTSD symptom severity in the multiple regression analysis, as hypothesized. The SRQ used in this study was phrased to ask about one specific person, the traumatized individual's close other participating in the larger study with them. To the author's knowledge, no studies have included the SRQ and SAQ simulatenously to explore their relative association with PTSD severity. The construct of social acknowledgment differs from the SRQ measure because it does not relate to the structural supportiveness or the functional supportiveness of the direct environment, but rather to a sense of having one's traumatic experience acknowledged and valued by members of one's social network and society at large. It may be possible that negative reactions to trauma disclosure from just one person are not as integral to PTSD symptom severity as a general feeling of lack of acknowledgment from different and multiple sources (i.e., society at large, family, and friends). Individuals in the current study may have selected a close other who they view as more supportive when completing the SRQ. The majority of participants had a close other participating in the larger study with them and the action of their close other participating in a research study 
with them may have made respondents more inclined to report fewer negative social interactions on the SRQ than they would have in other studies that did not include close others.

Also as expected, negative social interaction scales were positively correlated with each other and negatively correlated with measures of positive social support. This finding is in line with past research that suggests that these consructs are related to each other (Wagner et al., 2016) and supports Wagner et al.'s suggestion that social support exists on a continuum from negative to positively valenced interactions.

\section{Moderators of Relationships between Social Interactions and PTSD: Aim 2}

Contrary to expectation, the relationships between negative social interactions and PTSD symptoms were not moderated by sex, trauma type, or disclosure style as measured by DTQ Total scores. Based on the limited literature available on social factors in trauma recovery, Wagner et al. (2016) proposed these variables as potential moderators of the relationships between social interactions and PTSD severity. This finding suggests that regardless of sex, experiencing sexual interpersonal violence, nonsexual interpersonal or another type of trauma, or dysfunctional disclosure style, the relationship between negative social interactions and PTSD severity still holds. In other words, for both women and men, survivors of diverse traumas, and those with different trauma disclosure styles, lack of acknowledgment as a victim in society and disapproval from family and friends predicted PTSD symptoms.

Although the relationship between negative social interactions and PTSD symptoms was expected to be stronger for females, this was the first empirical study to explore sex as a moderator in the relationship between the SAQ, a measure of lack of 
societal acknowledgment as a victim, and PTSD symptom severity. The hypothesis that sex would moderate these relationships was based on past meta-analytic findings suggesting that females are at greater risk for PTSD (Brewin et al., 2000) than males, and results from Andrews et al. (2003) that women were more likely to report negative responses from others following trauma. However, Andrews and colleagues measured negative responses using only a single item and in their meta-analytic study, Brewin and colleagues did not find sex to moderate the relationships between social support and PTSD. DeLong (2013) also found that women reported fewer positive social interactions, and had significantly less support available compared with men. However, again, DeLong used different measures of social interactions than those in the current study and did not explore sex as a moderator of the relationship between PTSD and social interactions. Therefore, the hypothesis was formed on a small body of literature and the relationship between sex and social interactions to PTSD symptoms should continue to be explored.

In the current study, trauma type was categorized into sexual interpersonal violence, nonsexual interpersonal violence, and other traumas. Contrary to expectation, the relationships between general societal disapproval and family and friends disapproval with PTSD symptom severity did not differ according to type of trauma experienced. DeLong (2013) conducted the only other known empirical study to explore whether survivors of different types of trauma reported more negative interactions than others and found that childhood sexual abuse was associated with greater reports of negative social reactions. In the current study, participants were all adults (above age 18) who had been 
exposed to a traumatic event within the past 6 months and no participants reported their index trauma as childhood sexual assault.

Interestingly, in both models exploring trauma type as a moderator of the relationship between negative social interactions and PTSD, age emerged as a significant predictor of PTSD symptoms. Although prior history of trauma (e.g., exposure to trauma in childhood, length of time since a trauma prior to the index event that brought participants into the study) was not explored in the current study, age may be naturally associated with chronicity of PTSD symptoms when there is developmental trauma. Thus, there may be a stronger relationship between childhood sexual trauma and PTSD symptom severity compared with other types of trauma (Brewin et al., 2000). Yet, the finding that, regardless of the type of trauma experienced, negative social interactions were still related to PTSD symptom severity has important implications. This result implies that survivors of a range of traumas (e.g., accidents, natural disasters, witnessing suicide, physical abuse) endorse negative social interactions (e.g., Delong, 2013; Maercker \& Mueller, 2004) and supports Wagner et al.'s (2016) call for research in this area to expand beyond sexual assault survivors to explore the effects of negative and positive social interactions across samples of survivors of diverse traumas.

The finding that dysfunctional disclosure style did not moderate the relationship between general societal disapproval and family and friends disapproval was not expected. This was the first known study to use validated measures of disclosure style, negative social interactions, and clinician-administered PTSD assessment to explore dysfunctional disclosure style as a moderator in the relationship between PTSD severity and social interactions. One previous study examined aspects of disclosure as a 
moderator of the relationship between social support and PTSD (i.e., Balderrama-Durbin et al., 2013) and found that, within a sample of U.S. Air Force service members and their partners, willingness to disclose about their trauma did moderate this relationship. However, this study did not use a validated measure of disclosure style and only examined measures of positive social interactions, not negative ones. In studies of the SRQ and SAQ, negative social interactions are positively related to dysfunctional disclosure style (e.g., Maercker et al., 2009; Mueller et al., 2008; Ullman \& Filipas, 2005). These studies examined correlations between negative social interaction scales and measures of PTSD, but did not explore the DTQ as a moderator in these relationships. In accordance with these studies, in the current study, all scales measuring social interactions, except for the SAQ Recognition scale, had significant positive correlations with the DTQ Total score, suggesting there is a relationship between disclosure style and social interactions.

Consistent with past research (Maercker et al., 2009; Mueller et al., 2008; Ullman \& Filipas, 2005), DTQ Total scores were also significantly positively correlated with PTSD symptom severity. However, in the models exploring dysfunctional disclosure style as a moderator in the relationship between negative social interactions and PTSD symptom severity, the DTQ Total scale scores did not emerge as significant predictors of PTSD. It is possible that dysfunctional disclosure style did not moderate the relationships between negative social interactions and PTSD severity because of the timing of assessment. For example, participants in this study had been exposed to a traumatic event within the past 6 months and perhaps had just started the trauma disclosure process. As time elapses, individuals who experience greater PTSD symptoms and more negative 
social interactions may develop more dysfunctional disclosure styles (e.g., reluctance to talk, emotional reactions when disclosing) or those who continue to experience PTSD symptoms over time may engage in more dysfunctional disclosure behaviours as compared with those whose symptoms remit. Interestingly, scores on the DTQ were lower than those found in studies of samples of trauma survivors whose traumas were less recent. For example, in Maercker et al.'s (2009) study of 61 Chechens who had been refugees for 5 to 7 years (75\% estimated to have PTSD), DTQ Total scores $(M=94.48$, $S D=23.71$ ) were roughly 30 points higher than those in the current sample. The highest possible DTQ Total score is 150 and in the current sample, scores were on the lower end of the range, suggesting less dysfunctional disclosure style.

\section{Clinical Implications}

The current findings are in support of Wagner et al.'s (2016) conclusion that positive aspects of social interactions are less important in terms of prevention of PTSD than negative aspects of social interactions. In accordance with past researchers (e.g., Guay, Billette, \& Marchand, 2006; Wagner et al., 2016), these findings buttress the notion that early interventions to prevent PTSD should focus on mitigating negative social interactions. Although positive interactions may have some beneficial effects (as

general social support and recognition as a survivor had significant bivariate correlations with PTSD symptom severity), results of the current study point that efforts may be best directed at preventing and reducing the adverse effects of negative social interactions.

Clinicians can use results of the current study to educate trauma survivors about their interpretations of ambiguous social interactions and behaviours they engage in that may elicit negative interactions (e.g., avoidance of social situations, lack of participation 
in pleasurable social activities). For instance, if distorted, clinicians can teach cognitive modification strategies to help survivors understand how their perceptions of ambiguous social interactions may be negatively distorted or how their behaviours may contribute to negative interactions. Cognitive-behavioural interventions that target thought distortions and behaviours in both individual (e.g., Cognitive Processing Therapy; Resick, Monson, \& Chard, 2007) and couple-based treatments (e.g., Cognitive-Behavioral Conjoint Therapy; Monson \& Fredman, 2012; Monson et al., 2012) for PTSD have been found to be efficacious. These interventions are delivered after the onset of PTSD, but similar preventive interventions can be developed and delivered early on following trauma exposure to target maladaptive cognitions related to social experiences.

Perceptions of general societal disapproval emerged as the strongest contributor to PTSD symptoms, suggesting that interventions should target survivors' experiences with multiple social contacts rather than be narrowly focused on one support provider. Although interventions could be dyadic (e.g., Billette, Guay, \& Marchand, 2008; involving the trauma survivor and their close other), they should also focus on social experiences with one's wider network such as family, friends, colleagues, clergy, and experiences in society in general (e.g., interpretations of news reports, advertisements). Current dyadic interventions (e.g., Cognitive-Behavioural Conjoint Therapy) are focused on improving PTSD symptoms and relationship functioning among dyads where one individual has PTSD. However, these interventions could be broadened to teach couples strategies for addressing the trauma survivor's wider social experiences and communication with a broader network of social contacts. These early interventions could be aimed at modifying trauma survivors' perceptions of their social experiences at 
a broad level (e.g., thoughts that they are no longer a normal member of society, beliefs that no one can understand what they went through).

The finding that disapproval from family and friends was a significant predictor of PTSD again suggests that dyadic or family-based early interventions may be important. Indeed, interpersonally-oriented interventions have been found to be efficacious in both treating PTSD symptoms and enhancing social factors (e.g., Billette et al., 2008; Monson \& Fredman, 2012; Monson et al., 2012; Sautter, Glynn, Thompson, Franklin, \& Han, 2009), underscoring the importance of targeting social factors in preventive interventions for PTSD. Interventions that include family members could focus on communication skills to teach both trauma survivors and their loved ones how social interactions may be processed and their adverse effects. They can also provide psychoeducation about the importance of distorted thoughts and negative thinking to family and friends of survivors in order to increase understanding of how their actions may be interpreted by the trauma survivor.

The results have important implications about allocation of resources as they suggest that interventions should be developed, targeting both women and men and survivors of different traumas. Regardless of sex, trauma type or one's style of disclosing about their trauma, participants in the current study endorsed a range of both negative and positive social interactions. This highlights that clinicians should address the importance of social interactions in trauma recovery with a range of trauma survivors. As dysfunctional disclosure style did not moderate the relationship between negative social interactions and PTSD, disclosure style may not be an important factor to target in early interventions with trauma survivors. 
To the author's knowledge, one early intervention for preventing PTSD exists that targets social acknowledgment as a victim and disclosure of trauma in order to improve PTSD symptoms (Xu et al., 2015). This intervention is Web-based and uses five interactive modules to modify cognitive distortions about social support and their traumatic experiences and behaviours that may elicit negative social responses. The ultimate aim of this intervention is to enhance social support, improve disclosure of trauma style, and reduce perceptions of societal disapproval in order to improve PTSD symptoms. In a randomized controlled trial, Xu et al. (2015) found that among 21 individuals with PTSD symptoms for at least the past month, social acknowledgment (as measured by the SAQ) and disclosure of trauma (as measured by the DTQ) improved significantly after 1 month, and improvements on these measures mediated reduction in PTSD symptoms. Interventions, such Xu et al.'s Web-based program described above, should continue to be developed and tested given current findings of the importance of social acknowledgment as a victim on trauma recovery.

In addition, interventions such as the cognitive-behavioural therapy employed by Billette et al. (2008) and Cognitive-Behavioural Conjoint Therapy for PTSD (Monson \& Fredman, 2012) that include the trauma survivor's romantic partner should also continue to be explored. For example, Billette and colleagues found that including a spouse in the intervention enhanced social support and led to reductions in PTSD symptoms. These interventions could be expanded to not only focus on enhancing social support but to reduce negative interactions. 


\section{Limitations and Future Directions}

Although the current study had several strengths, a number of limitations should be noted. Participants were recruited from the Greater Toronto Area, limiting generalizability to trauma survivors across Canada and other countries. To be more representative of Canadians exposed to a traumatic event, future studies should employ stratified sampling based on age, trauma type (e.g., sexual assault, military combat, accident), sex, and ethnicity.

As described above, findings from the current study support the social negativity hypothesis. Theories such as the main- or direct-effects models and the stress-buffering hypothesis hold that positive interactions may buffer against the adverse mental health outcomes. It was not possible to recruit participants prior to trauma exposure, but such a design would enable better understanding of the potential buffering effects of positive social interactions in protecting against PTSD as well as establish whether negative interactions confer risk for PTSD. The current study used a cross-sectional design, assessing trauma survivors at one time point only (within 6 months) of trauma exposure, limiting conclusions related to the directionality of the relationship between negative social interactions and PTSD. In line with King et al.'s (2006) theory on erosion of social support, symptoms of PTSD may precipitate negative social interactions, rendering PTSD a risk factor for negative social interactions. Future studies should employ longitudinal designs to examine the directionality of relationships of social interactions and PTSD symptoms over time and follow individuals exposed to trauma as early as possible following exposure to determine the relative influence of positive and negative interactions acutely following trauma. Such findings would help to further clarify 
whether there are any buffering effects of positive support in the days and weeks following trauma exposure. It may be possible that positive interactions earlier on are protective against PTSD symptoms, but because of the design of the study, it was not possible to adequately test these theories.

Several factors such as comorbid mental health problems (e.g., depression, insomnia), an individual's history of social interactions prior to trauma exposure, quality of interpersonal relationships, personality features and cognitive styles could influence an individual's perceptions of posttraumatic interactions. For instance, individuals with depression appear to be at increased likelihood to recall negative social information as compared with nonpsychiatric and nondepressed control groups with other psychopathology (e.g., Gilboa-Schechtman et al., 2002). Poor sleep is also associated with a bias to recall ambiguous social events as negative (Barber \& Budnick, 2015).

The current study examined sex, disclosure style, and trauma type as moderators in the relationships between posttraumatic social interactions and PTSD symptoms, a novel contribution to the literature. However, these aforementioned other factors (e.g., comorbid depression or insomnia, quality of interpersonal relationships, personality features, cognitive style) may influence the relationship between social interactions and PTSD severity and were not explored. Future studies should explore the influence of these factors within relationships between social facets and PTSD symptoms. No studies to date have examined whether PTSD symptoms bias recall for negative over positive or neutral information. Experimental studies should examine whether PTSD diagnosis increases bias toward reporting negative social interactions. 
Along the same lines, in the moderation analyses, the covariate age was a significant predictor in models exploring the interactions between sex and general disapproval and family/friends disapproval, and in models exploring interactions between trauma type and these measures. Given the sample size, the current study was not adequately powered to examine the interaction between sex and age as well as trauma type and age and these social factors. However, it is possible that with increasing age, individuals are exposed to a greater number of traumatic events, or that for different sexes as age increases there is a higher likelihood that negative social interactions relate to PTSD symptom severity. For example, Brewin et al.'s (2000) meta-analysis found that in studies of PTSD among men, there were larger effect sizes for younger age at trauma. Brewin et al. (2000) also found that women were more likely than men to develop PTSD following childhood trauma. One possible explanation may be that the relationship between social acknowledgment as a victim and PTSD severity may be stronger for women exposed to traumas such as childhood sexual assault as compared with males. Although not possible to explore, given the current sample size, the possibility that both sex and trauma type may interact and jointly moderate the association between social support and PTSD should be explored.

Trauma type was explored as a moderator of the relationship between social interactions and PTSD symptom severity and trauma type was captured on the CAPS, and further categorized by the researcher into one of three types (i.e., nonsexual interpersonal violence, sexual interpersonal violence and other trauma type) due to the sample size and moderation analysis used. Perhaps the way trauma type was measured in the current study did not capture the important aspects of the event or severity of the 
trauma. Trauma type could have been measured in multiple ways, such as by assessing trauma severity with a validated measure or categorized differently, such as the Life Events Checklist (Gray, Litz, Hsu, \& Lombardo, 2004) or by using different categories. Despite its limitations, this study offers novel findings on the importance of different social facets in relation to trauma recovery in participants exposed to a recent trauma event. Among survivors of various traumatic events, perceptions of disapproval from society in general and from family and friends appear to be related to recovery from trauma. In contrast, supportive interactions may not be protective against PTSD when considered in conjunction with these negative interactions and may be less important to target in interventions. Early interventions should concentrate on mitigating the adverse effects of negative social interactions, rather than solely enhancing social support. This study has important clinical implications and suggests areas for future study in order to advance understanding of the role of social interactions in trauma recovery. 


\section{References}

American Psychiatric Association. (2000). Diagnostic and statistical manual of mental disorders: DSM-IV-TR. Washington, DC: American Psychiatric Association.

American Psychiatric Association. (2013). Diagnostic and statistical manual of mental disorders (5th edition). Arlington, VA: American Psychiatric Association.

Andrews, B., Brewin, C. R., \& Rose, S. (2003). Gender, social support, and PTSD in victims of violent crime. Journal of Traumatic Stress, 16, 421-427. doi: 10.1023/A:1024478305142

Baker, T., Skolnik, L., Davis, R., \& Brickman, E. (1991). The social support of survivors of rape: The differences between rape survivors and survivors of other violent crimes and between husbands, boyfriends, and women friends. In Rape and sexual assault III (Garland Pu). New York, NY.

Balderrama-Durbin, C., Snyder, D. K., Cigrang, J., Talcott, G. W., Tatum, J., Baker, M., ... Smith Slep, A. M. (2013). Combat disclosure in intimate relationships: Mediating the impact of partner support on posttraumatic stress. Journal of Family Psychology, 27, 560-568. doi: 10.1037/a0033412

Barber, L. K., \& Budnick, C. J. (2015). Turning molehills into mountains: Sleepiness increases workplace interpretive bias. Journal of Organizational Behavior, 36, 360-381. doi:10.1002/job.1992

Billette, V., Guay, S., \& Marchand, A. (2008). Posttraumatic stress disorder and social support in female victims of sexual assault: The impact of spousal involvement on the efficacy of cognitive-behavioral therapy. Behavior Modification, 32, 876-896. 
doi: $10.1177 / 0145445508319280$

Blake, D. D., Weathers, F. W., Nagy, L. M., Kaloupek, D. G., Gusman, F. D., Charney, D. S., \& Keane, T. M. (1995). The development of a clinician-administered PTSD scale. Journal of Traumatic Stress, 8, 75-90. doi: 10.1002/jts.2490080106

Brewin, C. R., Andrews, B., \& Valentine, J. D. (2000). Meta-analysis of risk factors for posttraumatic stress disorder in trauma-exposed adults. Journal of Consulting and Clinical Psychology, 68, 748-766. doi: 10.1037/0022-006X.68.5.748

Charuvastra, A., \& Cloitre, M. (2008). Social bonds and posttraumatic stress disorder. Annual Review of Psychology, 59, 301-328. doi:

10.1146/annurev.psych.58.110405.085650

Cohen, J., \& Mannarino, A. P. (2008). Disseminating and implementing trauma-focused CBT in community settings. Trauma, Violence \& Abuse, 9, 214-226. doi: $10.1177 / 1524838008324336$

Cohen, S., \& Wills, T. A. (1985). Stress, social support, and the buffering hypothesis. Psychological Bulletin, 98, 310-357. doi: 10.1037/0033-2909.98.2.310

Davis, R. C., \& Brickman, E. (1996). Supportive and unsupportive aspects of the behavior of others toward victims of sexual and nonsexual assault. Journal of Interpersonal Violence, 11, 250-262. doi: 10.1177/088626096011002008

Davis, R. C., Brickman, E., \& Baker, T. (1991). Supportive and unsupportive responses of others to rape victims: Effects on concurrent victim adjustment. American Journal of Community Psychology, 19, 443-451. doi: 10.1007/BF00938035

DeLong, H. (2013). Social support in PTSD: An analysis of gender, race, and trauma 
type. Discussions, 8, 1-3. doi: Retrieved from

http://www.studentpulse.com/a?id=802

Dunmore, E., Clark, D. M., \& Ehlers, A. (2001). A prospective investigation of the role of cognitive factors in persistent posttraumatic stress disorder (PTSD) after physical or sexual assault. Behaviour Research and Therapy, 39, 1063-1084. doi: 10.1016/S0005-7967(00)00088-7

Ehlers, A., Mayou, R. A., \& Bryant, B. (1998). Psychological predictors of chronic posttraumatic stress disorder. Journal of Abnormal Psychology, 107, 508-519. doi: 10.1037/0021-843X.107.3.508

Fankhauser, S., Wagner, B., Krammer, S., Aeschbach, M., Pepe, A., Maercker, A., \& Forstmeier, S. (2011). The impact of social and interpersonal resources on adjustment disorder symptoms in older age. GeroPsych, 23, 227-241. doi: $10.1024 / 1662-9647 / \mathrm{a} 000022$

Guay, S., Billette, V., \& Marchand, A. (2006). Exploring the links between posttraumatic stress disorder and social support: Processes and potential research avenues. Journal of Traumatic Stress, 19, 327-338. doi: 10.1002/jts.20124

Gilboa-Schectman, E., Erhard-Weiss, D., \& Jeczemien, P. (2002). Interpersonal deficits meet cognitive biases: Memory for facial expressions in depressed and anxious men and women. Psychiatry Research, 113, 279-293. doi: 10.1016/S0165$1781(02) 00266-4$

Guay, S., Beaulieu-Prévost, D., Beaudoin, C., St-Jean-Trudel, É., Nachar, N., Marchand, A., \& O'connor, K. P. (2011). How do social interactions with a significant other 
affect PTSD Symptoms? An empirical investigation with a clinical sample. Journal of Aggression, Maltreatment \& Trauma, 20, 280-303. doi:

$10.1080 / 10926771.2011 .562478$

Gray, M., Litz, B., Hsu, J., \& Lombardo, T. (2004). Psychometric properties of the Life Events Checklist. Assessment, 11, 330-341. doi: 10.1177/1073191104269954

Hayes, A. F. (2012). PROCESS: A versatile computational tool for observed variable mediation, moderation, and conditional process modeling [White paper]. Retrieved July 29, 2016, from http://www.afhayes.com/ public/process2012.pdf

Hayes, A. F. (2015). Hacking PROCESS to estimate a simple moderation model with a three-category moderator. [White paper]. Retrieved July 29, 2016, from http://afhayes.com/public/quadratichack.pdf

Helgeson, V. S. (2003). Social support and quality of life. Quality of Life Research, 12, 25-31. doi: 10.1023/A:1023509117524

Hobfoll, S. (1988). The ecology of stress. New York, NY: Hemisphere.

Hovens, J. E., Van der Ploeg, H. M., Klaarenbeek, M. T., Bramsen, I., Schreuder, J. N., \& Rivero, V. V. (1994). The assessment of posttraumatic stress disorder with the Clinician-Administered PTSD Scale: Dutch results. Journal of Clinical Psychology, 50, 325-340. doi: 10.1002/1097-4679(199405)50:3<325::AID-

JCLP2270500304>3.0.CO;2-M

IBM Corp. (2010). IBM SPSS Statistics for Windows, Version 19.0. Armonk, NY: IBM Corp.

Jones, B., Mueller, J., \& Maercker, A. (2006). Trauma and posttraumatic reactions in 
German development aid workers: prevalences and relationship to social acknowledgment. The International Journal of Social Psychiatry, 52, 91-100. doi: $10.1177 / 0020764006061248$

Kaniasty, K., \& Norris, F. H. (2004). Social support in the aftermath of disasters, catastrophes, and acts of terrorism: Altruistic, overwhelmed, uncertain, antagonistic, and patriotic communities. Bioterrorism: Psychological and Public Health Interventions, 200-229. doi: 10.1037//1089-2680.6.4.307

Kessler, R. C. (2000). Posttraumatic stress disorder: The burden to the individual and to society. Journal of Clinical Psychiatry, 61, 171-181. Retrieved from http://onlinelibrary.wiley.com/journal/10.1002/(ISSN)1097-4679

Kim, H. Y. (2013). Statistical notes for clinical researchers: Assessing normal distribution using skewness and kurtosis. Restorative Dentistry \& Endodontics, 38, 52-54. doi: 10.5395/rde.2013.38.1.52

King, D. W., Taft, C., King, L. A., Hammond, C., \& Stone, E. R. (2006). Directionality of the association netween social support and posttraumatic stress disorder: A longitudinal investigation. Journal of Applied Social Psychology, 36, 2980-2992. doi: 10.1111/j.0021-9029.2006.00138.x

Laffaye, C., Cavellla, S., Drescher, K., \& Rosen, C. (2008). Relationships among PTSD symptoms, social support, and support source in veterans with chronic PTSD. Journal of Traumatic Stress, 21, 394-401. doi: 10.1002/jts.20348 
Lakey, B., \& Cassady, P. B. (1990). Cognitive processes in perceived social support. Journal of Personality and Social Psychology, 59, 237-343. doi: 10.1037/00223514.59.2.337

Lecrubier, Y., Sheehan, D. V., Weiller, E., Amorim, P., Sheehan, K. H., Janavs, J., \& Dunbar, G. C. (1997). The Mini International Neuropsychiatric Interview (MINI). A short diagnostic structured interview: Reliability and validity according to the CIDI. European Psychiatry, 12, 224-231. doi: 10.1016/S0924-9338(97)83296-8

Littleton, H. L. (2010). The impact of social support and negative disclosure reactions on sexual assault victims: A cross-sectional and longitudinal investigation. Journal of Trauma \& Dissociation, 11, 210-227. doi: 10.1080/15299730903502946

Maercker, A., \& Mueller, J. (2004). Social acknowledgment as a victim or survivor: A scale to measure a recovery factor of PTSD. Journal of Traumatic Stress, 17, 345351. doi: 10.1023/B:JOTS.0000038484.15488.3d

Maercker, A., Povilonyte, M., Lianova, R., \& Pöhlmann, K. (2009). Is acknowledgment of trauma a protective factor? The sample case of refugees from Chechnya. European Psychologist, 14, 249-254. doi: 10.1027/1016-9040.14.3.249

Major, B., Zubek, J. M., Cooper, M. L., Cozzarelli, C., \& Richards, C. (1997). Mixed messages: Implications of social conflict and social support within close relationships for adjustment to a stressful life event. Journal of Personality and Social. doi: 10.1037//0022-3514.72.6.1349

Monson, C. M., \& Fredman, S. J. (2012). Cognitive-behavioral conjoint therapy for posttraumatic stress disorder. New York, NY: Guilford. 
Monson, C. M., Fredman, S. J., Macdonald, A., Pukay-Martin, N. D., Resick, P. A., \& Schnurr, P. P. (2012). Effect of Cognitive-Behavioral Couple Therapy for PTSD: A randomized controlled trial. The Journal of the American Medical Association, 308, 700-709. doi: 10.1001/jama.2012.9307

Mueller, J., Hanspeter, M., \& Maercker, A. (2008). Disclosure and social acknowledgment as predictors of recovery from posttraumatic stress: A longitudinal study in crime victims. The Canadian Journal of Psychiatry, 53, 160-168. Retrieved from http://search.proquest.com/docview/222799391?accountid=14483

Mueller, J., Beauducel, A., Raschka, J., \& Maercker, A. (2000).

Kommunikationsverhalten nach politischer Haft in der DDR. Entwicklung eines Fragebogens zum Offenlegen der Traumaerfahrungen. [Communicative styles after political imprisonment in the GDR: Development of a questionnaire regarding disclosure of traumatic e. Zeitschrift F“ur Politische Psychologie, 4, 413-427. Retrieved from http://amnesty-heilberufe.de/wp-content/uploads/2014/02/2001traumaerfahrung-kommunikation-ddr-Mueller.pdf

Mueser, K. T., Salyers, M. P., Rosenberg, S. D., Ford, J. D., Fox, L., \& Carty, P. (2001). Psychometric evaluation of trauma and posttraumatic stress disorder assessments in persons with severe mental illness. Psychological Assessment, 13, 110-117. doi: $10.1037 / 1040-3590.13 .1 .110$

Nietlisbach, G., \& Maercker, A. (2009). Social cognition and interpersonal impairments in trauma survivors with PTSD. Journal of Aggression, Maltreatment \& Trauma, 18, 382-402. doi: 10.1080/10926770902881489

Ozer, E. J., Best, S. R., Lipsey, T. L., \& Weiss, D. S. (2003). Predictors of posttraumatic 
stress disorder and symptoms in adults: A meta-analysis. Psychological Bulletin, 129, 52-73. doi: 10.1037/1942-9681.S.1.3

Pielmaier, L., \& Maercker, A. (2011). Psychological adaptation to life-threatening injury in dyads: The role of dysfunctional disclosure of trauma. European Journal of Psychotraumatology, 2, 1-12. doi: 10.3402/ejpt.v2i0.8749

Resick, P. A., Monson, C. M., \& Chard, K. M. (2007). Cognitive processing therapy: Veteran/military version. Washington, DC: Department of Veteran's Affairs.

Riggs, D. S., Rothbaum, B. O., \& Foa, E. B. (1995). A prospective examination of symptoms of posttraumatic stress disroder in victims of nonsexual assault. Journal of Interpersonal Violence, 10, 201-214. doi: 10.1177/0886260595010002005

Rook, K.S. (1984). The negative side of social interaction: Impact on psychological wellbeing. Journal of Personality and Social Psychology, 46, 1097-1108. doi: $10.1037 / 0022-3514.46 .5 .1097$

Rothbaum, B. O., Foa, E. B., Riggs, D. S., Murdock, T., \& Walsh, W. (1992). A prospective examination of posttraumatic stress disorder in rape victims. Journal of Traumatic Stress, 5, 455-475. doi: 10.1007/BF00977239

Sautter, F. J., Glynn, S. M., Thompson, K. E., Franklin, L., \& Han, X. (2009). A couplebased approach to the reduction of PTSD avoidance symptoms: Preliminary findings. Journal of Marital and Family Therapy, 35, 343-349. doi:10.1111/j.17520606.2009.00125.x

Sheehan, D. V., Lecrubier, Y., Sheehan, K. H., Amorim, P., Janavs, J., Weiller, E.,... Dunbar, G. C. (1998). The Mini-International Neuropsychiatric Interview (MINI): 
the development and validation of a structured diagnostic psychiatric interview for DSM-IV and ICD-10. Journal of Clinical Psychiatry, 59, 22-33. Retrieved from http://www.ncbi.nlm.nih.gov/pubmed/9881538

Solomon, Z., Mikulincer, M., \& Flum, H. (1989). The implications of life events and social integration in the course of combat-related posttraumatic stress disorder. Social Psychiatry and Psychiatric Epidemiology, 24, 41-48. doi:

\subsection{7/BF01788199}

Sutker, P. B., Davis, J. M., Uddo, M., \& Ditta, S. R. (1995). War zone stress, personal resources, and PTSD in Persian Gulf War returnees. Journal of Abnormal Psychology, 104, 444-452. doi: 10.1037/0021-843X.104.3.444

Tavakol, M., \& Dennick, R. (2011). Making sense of Cronbach's alpha. International Journal of Medical Education, 2, 53-55. doi: 10.5116/ijme.4dfb.8dfd

Turner, R. J., \& Marino, F. (1995). Social support and social structure: A descriptive epidemiology. Journal of Health and Social Behavior, 35, 193-212. Retreived from: http://www.jstor.org/stable/2137276

Ullman, S. E. (1999). Social support and recovery from sexual assault: A review. Aggression and Violent Behavior, 4, 343-358. doi: 10.1016/S1359-1789(98)000068

Ullman, S. E. (2000). Psychometric characteristics of the Social Reactions Questionnaire. Psychology of Women Quarterly, 24, 257-271. doi: 10.1111/j.14716402.2000.tb00208.x

Ullman, S. E. (2008). Relationship to perpetrator, disclosure, social reactions, and PTSD 
symptoms in child sexual abuse survivors. Health Psychology, 37-41. doi: 10.1300/J070v16n01

Ullman, S. E., \& Filipas, H. H. (2001). Predictors of PTSD symptom severity and social reactions in sexual assault victims. Journal of Traumatic Stress, 14, 369-389. doi: 10.1023/A:1011125220522

Ullman, S. E., \& Filipas, H. H. (2005). Gender differences in social reactions to abuse disclosures, postabuse coping, and PTSD of child sexual abuse survivors. Child Abuse and Neglect, 29, 767-782. doi: 10.1016/j.chiabu.2005.01.005

Ullman, S. E., Filipas, H. H., Townsend, S. M., \& Starzynski, L. L. (2006). The role of victim-offender relationship in women's sexual assault experiences. Journal of Interpersonal Violence, 21, 798-819. doi: 10.1177/0886260506288590

Ullman, S. E., Filipas, H. H., Townsend, S. M., \& Starzynski, L. L. (2007). Psychosocial correlates of PTSD symptom severity in sexual assault survivors. Journal of Traumatic Stress, 20, 821-831. doi: 10.1002/jts.20290

Ullman, S. E., Starzynski, L. L., Long, S. M., Mason, G. E., \& Long, L. M. (2008). Exploring the relationships of women's sexual assault disclosure, social reactions, and problem drinking. Journal of Interpersonal Violence, 23, 1235-1257. doi: $10.1177 / 0886260508314298$

Ullman, S. E., Townsend, S. M., Filipas, H. H., \& Starzynski, L. L. (2007). Structural models of the relations of assault severity, social support, avoidance coping, selfblame, and PTSD among sexual assault survivors. Psychology of Women Quarterly, 31, 23-37. doi: 10.1111/j.1471-6402.2007.00328.x 
Van Ameringen, M., Mancini, C., Patterson, B., \& Boyle, M. H. (2008). Posttraumatic stress disorder in Canada. CNS Neuroscience \& Therapeutics, 14, 171-181. doi: 10.1111/j.1755-5949.2008.00049.x

Wagner, B., Keller, V., Knaevelsrud, C., \& Maercker, A. (2012). Social acknowledgment as a predictor of posttraumatic stress and complicated grief after witnessing assisted suicide. International Journal of Social Psychiatry, 58, 381-385. doi: $10.1177 / 0020764011400791$

Wagner, A. C., Monson, C. M., Hart, T. L. (2016). Understanding social factors in the context of trauma: Implications for measurement and intervention. Journal of Aggression, Maltreatment \& Trauma, Advance online publication. Retrieved from http://dx.doi.org/10.1080/10926771.2016.1152341

Weathers, F. W., Keane, T. M., \& Davidson, J. R. T. (2001). Clinician-administered PTSD scale: A review of the first ten years of research. Depression and Anxiety, 13, 132-156. doi: 10.1111/j.1365-2036.2011.04753.x

Weidmann, A., Fehm, L., \& Fydrich, T. (2008). Covering the tsunami disaster: Subsequent posttraumatic and depressive symptoms and associated social factors. Stress and Health, 24, 129-135. doi: 10.1002/smi.1168

Weiss, L. G. (2008). Toward the mastery of resiliency. Canadian Journal of School Psychology, 23, 127-137. doi: 10.1177/0829573508316600

Wolfe, J., Sharkansky, E. J., Read, J. P., Dawson, R., Martin, J. A., \& Ouimette, P. C. (1998). Sexual harrassment and assault as predictors of PTSD symptomatology among U.S. female Persian Gulf war military personnel. Journal of Interpersonal 
Violence, 13, 40-57. doi: 10.1177/088626098013001003

Xu, W., Wang, J., Wang, Z., Li, Y., Yu, W., Xie, Q., ... Maercker, a. (2015). Web-based intervention improves social acknowledgment and disclosure of trauma, leading to a reduction in posttraumatic stress disorder symptoms. Journal of Health Psychology. doi: $10.1177 / 1359105315583371$ 